\title{
Dust-enshrouded star near supermassive black hole: predictions for high-eccentricity passages near low-luminosity galactic nuclei
}

\author{
Michal Zajaček ${ }^{1,2}$, Vladimír Karas ${ }^{1}$, and Andreas Eckart ${ }^{3,4}$
}

\author{
1 Astronomical Institute, Academy of Sciences, Boční II 1401, 14100 Prague, Czech Republic \\ e-mail: michal_zajacek@yahoo.com \\ 2 Charles University in Prague, Faculty of Mathematics and Physics, V Holešovičkách 2, 18000 Prague, Czech Republic \\ 3 I. Physikalisches Institut der Universität zu Köln, Zülpicher Strasse 77, 50937 Köln, Germany \\ ${ }^{4}$ Max-Planck-Institut für Radioastronomie (MPIfR), Auf dem Hügel 69, 53121 Bonn, Germany \\ Received 19 September 2013 / Accepted 18 March 2014
}

\section{ABSTRACT}

\begin{abstract}
Context. Supermassive black holes reside in cores of galaxies, where they are often surrounded by a nuclear cluster and a clumpy torus of gas and dust. Mutual interactions can set some stars on a plunging trajectory towards the black hole.

Aims. We model the pericentre passage of a dust-enshrouded star during which the dusty envelope becomes stretched by tidal forces and is affected by the interaction with the surrounding medium. In particular, we explore under which conditions these encounters can lead to periods of enhanced accretion activity.

Methods. We discuss different scenarios for such a dusty source. To this end, we employed a modification of the Swift integration package. Elements of the cloud were modelled as numerical particles that represent the dust component that interacts with the optically thin gaseous environment.

Results. We determine the fraction of the total mass of the dust component that is diverted from the original path during the passages through the pericentre at $\simeq 10^{3}$ Schwarzschild radii and find that the main part of the dust ( $\gtrsim 90 \%$ of its mass) is significantly affected upon the first crossing. The fraction of mass captured at the second passage generally decreases to very low values.

Conclusions. As an example, we show predictions for the dusty source evolution assuming the current orbital parameters of the G2 cloud (also known as Dusty S-Cluster Object, DSO) in our Galactic centre. Encounter of a core-less cloud with a supermassive black hole is, most likely, a non-repeating event: the cloud is destroyed. However, in the case of a dust-enshrouded star, part of the envelope survives the pericentre passage. We discuss an offset of $\lesssim 0.3 \operatorname{arcsec}$ between the centre of mass of the diverted part and the star along the eccentric orbit. Finally, we examine an interesting possibility of a binary star embedded within a common wind envelope that becomes dispersed at the pericentre passage.
\end{abstract}

Key words. black hole physics - Galaxy: center - galaxies: individual: Sgr A*

\section{Introduction}

Most galaxies host supermassive black holes (SMBH; $10^{6} M_{\odot} \lesssim$ $M_{\bullet} \lesssim 10^{9} M_{\odot}$ ) in their cores, where these accrete gas and dust in the form of an accretion flow from their immediate neighbourhood (Krolik 1999; Meier 2012). The example nearest to us is the compact radio source $\mathrm{Sgr} \mathrm{A}^{*}$, which contains a black hole of mass $M_{\bullet}=4.4 \times 10^{6} M_{\odot}$ at distance $8.2 \mathrm{kpc}$ in the centre of the Milky Way (Eckart et al. 2005; Melia 2007; Genzel et al. 2010).

The character of accretion and the corresponding accretion rate vary greatly over different galaxy types. It appears that the availability of mass supply and the accretion mode that is established in the course of evolution of the system are the main agents that determine the power output and the spectral energy distribution of supermassive black holes (Frank et al. 2002). In several ways, the Galactic centre can serve as a paradigm for low-luminosity nuclei.

Active galactic nuclei (AGN) and quasars host radiatively efficient types of disc accretion (i.e., the standard scheme of geometrically thin accretion discs, or slim discs; Shakura \& Sunyaev 1973; Abramowicz et al. 1988) with accretion rates reaching and even exceeding the Eddington limit of $\dot{M}_{\text {Edd }} \simeq L_{\text {Edd }} /\left(0.1 c^{2}\right)$, where

$L_{\mathrm{Edd}}=\frac{4 \pi G M_{\bullet} m_{\mathrm{p}} c}{\sigma_{\mathrm{T}}} \simeq 1.3 \times 10^{44} \frac{M_{\bullet}}{10^{6} M_{\odot}} \quad[\mathrm{erg} / \mathrm{s}]$,

with $m_{\mathrm{p}}$ proton mass, $\sigma_{\mathrm{T}}$ Thomson cross-section.
Low-luminosity nuclei exhibit significantly lower accretion rates, $\dot{M}_{\bullet} \ll \dot{M}_{\text {Edd }}$ (Eckart et al. 2013a). This can be explained as a combination of a diminishing supply of material falling onto the black hole and the radiatively inefficient mode of accretion at certain stages. In this context, the present state of the Galactic centre represents an extreme example of an inactive nucleus: $\dot{M}_{\bullet} \simeq 10^{-8} M_{\odot}$ per year, which can be understood in terms of advection-dominated flow (Narayan \& McClintock 2008) ${ }^{1}$.

The temperature of the accreted material grows in the course of its infall in the gravitational field of the central black hole because the potential energy is converted into heat and is only partially released in the form of emerging radiation (e.g. Frank et al. 2002; Krolik 1999). While at the distance of several tens to hundreds Schwarzschild radii $\left(r_{\mathrm{s}} \equiv 2 G M_{\bullet} / c^{2} \doteq 2.95 \times\right.$ $\left.10^{5} M_{\bullet} / M_{\odot} \mathrm{cm}\right)$ the medium consists of ionised gas of the accretion disc and hot, diluted corona, farther out the temperature drops below the critical value for dust sublimation, $T_{\text {sub }} \simeq$ $1.5 \times 10^{3} \mathrm{~K}$ (Barvainis 1987; Krishna Swamy 2005). Therefore,

\footnotetext{
For the supermassive black hole of Sgr A* in the Galactic centre, the quiescent bolometric luminosity is $L_{\text {bol }}=\eta \dot{M} \cdot c^{2} \simeq 10^{36} \mathrm{erg} \mathrm{s}^{-1}$. This corresponds to the dimensionless efficiency parameter for the conversion of accreted mass into radiation of about $\eta \simeq 10^{-3}$, although it can be as low as $10^{-5}$ at the present stage of the source. The accretion outflow of Sgr A* is radiatively inefficient compared with predictions from the standard accretion disc theory, where $\eta \simeq 0.06-0.42$ is the predicted range.
} 
at larger distances a clumpy torus can persist with a fraction of its mass in the form of dust (Krolik \& Begelman 1988; Hönig \& Kishimoto 2010).

An equilibrium can be reached through processes of dust sublimation (by strong irradiation from the central source and stars of the nuclear cluster), in competition with the replenishment of dust by stellar winds and the infall of clouds from the outer regions, where the circumnuclear torus is present (Antonucci 1993; Urry \& Padovani 1995). The co-evolution of gas and dust phases within clouds falling onto a supermassive black hole is relevant for our understanding of mass transport in the innermost regions of galactic nuclei.

Recently, an infrared-excess source named G2/DSO has been discovered (Gillessen et al. 2012) and subsequently detected in $L$ - and $K$-bands (Gillessen et al. 2013a; Phifer et al. 2013; Eckart et al. 2013b). It may indeed be a manifestation of a common mechanism of material transport in low-luminosity nuclei. We analyse the scenario of an infrared-excess, dusty stellar source. As indicated in Shcherbakov (2014), the cloud component of the source is optically thin and diluted and not thick and dense. Therefore, it is valid to assume that the cloud component is mainly constituted by the gaseous wind driven by the radiation pressure of central star and the dust that is located and formed in such a wind. This is the reason that in the following analysis we assume the dust to be in contact only with stellar wind and the ambient atmosphere around $\mathrm{Sgr}$ A* through which G2/DSO travels.

The adopted scenario is not necessarily only connected to this single event. It may be applied to other observed infraredexcess stellar sources that have been shown to move through the gaseous medium near the Galactic centre (e.g., Moultaka et al. 2005; Mužić et al. 2010). Moreover, it may be relevant for modelling the environment in other low-luminosity active galactic nuclei.

In this paper we adopt a simplified (toy) model: dust grains are treated as numerical particles under the influence of gravity of SMBH $\left(M=M_{\bullet}\right)$ and the embedded star $\left(M=M_{\star}\right)$, or the components of a binary $\left(M_{\star}^{(1)}, M_{\star}^{(2)}\right)$, and the effect of an outflowing wind of gas. We focus on dust-enshrouded stars with different distributions of dust bound to the central star, and we explore the amount of material that is lost from the cloud to the black hole. Effects arise from the ambient pressure of a central wind, the wind pressure from the star, and a bow-shock forming at the interface of winds. The star moves at transonic speed near the pericentre.

In this way we address the question whether and how dust particles embedded in the wind envelope are affected by close passages near the SMBH. For most model parameters, most of the dusty material is stripped from the envelope already on the first transit. For low accretion rates, the dust component can survive down to quite small radii, especially in regions shielded by obscuration. Furthermore, if the Field criterion (Field 1965; Barai et al. 2012) is fulfilled for the thermal stability of a twotemperature medium, the dust may co-exist with the hot medium at the same radius. The dust component by itself would be unimportant, but it contributes significantly to the radiation in NIR and tracing it helps to understand the observed emission. Similar treatment of dust dynamics is often employed in other astrophysical systems (mainly protoplanetary discs and stellar atmospheres, see e.g., van Marle et al. 2011).

We model the encounters over a broad span of parameters. To present specific examples we use orbital parameters relevant for the Galactic centre G2/DSO infrared source (Gillessen et al. 2013b), and we also attempt to distinguish among different outcomes of the passages through the pericentre (Eckart et al. 2013c; Phifer et al. 2013).

Furthermore, we point out to the possibility that the stellar core may actually consist of two components of a binary star. This idea is suggested by models of the origin of S-stars in the Galactic centre as a product of three-body interaction during the pericentre passage of a binary star on a highly eccentric trajectory (Gould \& Quillen 2003; Perets et al. 2007). Although the presence of a stellar core and its putative binary nature within the G2 cloud are on a purely hypothetical level, this scenario can connect, in a natural way, two apparently different aspects: the high eccentricity of the plunging trajectory, and the origin of the population of stars near the supermassive black hole. If there is indeed a star enshrouded by a dusty atmosphere, it was proposed that high eccentricity can be achieved by the Kozai mechanism (Šubr \& Karas 2005) or by resonant relaxation (Hopman \& Alexander 2006).

The geometrical setup and the main ingredients of our model are illustrated in Fig. 1. Three different flavours of the basic scenario were considered: a core-less cloud infalling onto the $\mathrm{SMBH}$, a star embedded within the dusty envelope, and an embedded binary that becomes disrupted near the SMBH. We focus on the latter two scenarios. Our simplified approach is complementary to purely hydrodynamical situations that neglect the dust component (Anninos et al. 2012; Burkert et al. 2012; Ballone et al. 2013; Shcherbakov 2014), which is consistent with an optically thick, dense medium where the dust is dragged along with the gas. However, for optically thin atmospheres, dust dynamics needs to be treated separately.

In the following analysis, we do not treat $\mathrm{Br} \gamma$ production or the radiation processes in the bow-shock region (Sacowski et al. 2013). We do see, however, that the dusty envelope is stretched by the gravitational and drag forces (depending on the initial distribution of particles in phase space and the parameters of the wind outflow), which leads to the gradual offset between the dust component and the stellar core. We note that observationally any difference between the cloud location in $L$-band with respect to the location of $\mathrm{K}(\mathrm{Br} \gamma)$ in the orbit is most likely due to uncertainties in the determination of the orbital positions; it may be heavily affected by different systematics in the two bands.

The paper is organized as follows: in Sect. 2 we set up the model and describe the numerical procedure to explore the mutual interaction between the star and its environment. We discuss the dependence of the dust temperature on the distance and the luminosity of the central source. Then we consider the effect of the star enshrouded by an initially spherical dusty envelope and a remnant disc. In Sect. 3, we present the results of the simulations including the wind blowing from the centre and the effect of the bow-shock region. We compare the difference between a disc-like Keplerian distribution and a Gaussian distribution of particles in the phase space. Finally, we determine the fraction of dust mass affected at subsequent encounters, and we show the offset that develops gradually between the centre of mass of the cloud and the nominal position of the star in the orbit. In Sect. 4 we summarize and discuss our results, and we conclude in Sect. 5.

\section{Model setup, numerical scheme, and tests}

\subsection{Clouds infalling onto $S M B H$ - test runs}

Accretion tori are not smooth, instead, they often form individual clouds with a mixed composition of gas and dust phases (e.g., Cox 2005; Vladimirov et al. 2005). The patchy structure evolves 

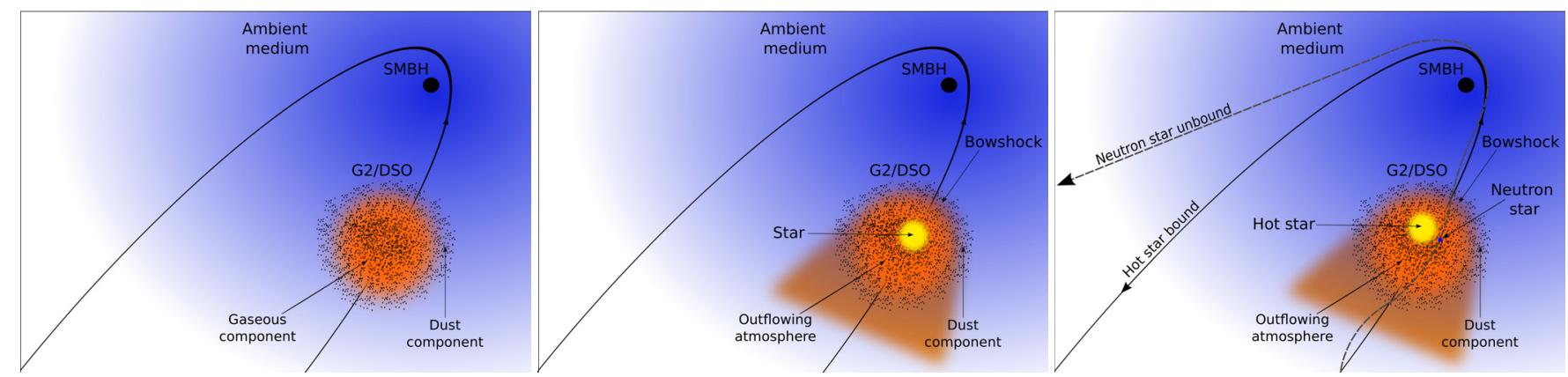

Fig. 1. Three variants of the model setup for which the predictions are qualitatively different especially at the post-pericentre phase. The model ingredients include the central supermassive black hole (black circle), an infalling cloud made of gas and dust (red), an embedded star (yellow), and a hot diluted flow (blue). We consider the pericentre at about $10^{3}$ Schwarzschild radii, so that the star is not expected to be tidally disrupted. However, the gaseous/dusty envelope is affected significantly. Left panel: a core-less cloud on an eccentric trajectory, interacting with the diluted ambient medium near the SMBH. Middle panel: the cloud enshrouds an embedded star. A radial wind of the outflowing gaseous atmosphere occurs and a bow-shock forms ahead of the stellar body. Right panel: binary system enclosed by a common envelope that becomes largely dispersed at the first pericentre passage. At the same time, the three-body interaction with the central SMBH causes the binary components to separate from the nominal trajectory.

by collisions, and some of the clumps can be set on a highly eccentric trajectory with the pericentre close to the central black hole. Interaction with the surrounding environment leads to the drift and gradual separation of different constituents of the cloud and deformation of its shape - dust and gas components of the cloud move with respect to each other, and with respect to the star (if embedded in the cloud core).

A bow shock develops around an embedded star and different species are transported across discontinuities in a different manner (smaller grains tend to be bound to the gas component, while the large grains are capable of penetrating into the interstellar medium; cf. MacGregor \& Stencel 1992; van Marle et al. 2011; Keppens et al. 2012). If large portions of the medium are neutral, viscous forces play an important role, while in a fully ionised plasma the efficient mechanism of dust grain charging operates. Then the main parameter that defines the coupling between dust and gas is the Coulomb coupling parameter, which is the ratio of the Coulomb potential energy of the particle interaction to the kinetic energy of the thermal motion. Depending on the system parameters, both weak coupling and strong coupling have been observed in dusty plasmas.

The grain size is a dominant factor for the forces acting on the gas/dust mixture. The mutual coupling between these two components of the complex plasma (including the viscous forces) is thought to just increase the effective dust grain mass by the amount of gas that is dragged along in the coupling. The particle mass is not relevant within the strong gravitational field of the SMBH and/or the star, instead, it may only be relevant for the wind force acting on the dust. For a gas-to-dust ratio of 100:1, the effective increase of the grain mass load by two orders of magnitude would then correspond to the grain size variation by factor of about 5 . This is well contained in the factor of a hundred in the grain sizes that we considered here. The relation between size and mass is uncertain because grains are most likely fluffy and not solid. Moreover, other factors are also connected with the grain size, for example, the typical electric charge that can develop by the interaction with the surrounding plasma and by photoionisation.

An infalling cloud passes through the external environment with physical properties spanning a wide range of values. We modelled the passages of stars with dusty envelopes through the pericentre by numerically integrating the trajectories of star(s) and that of dust particles that represent one of elementary constituents of the cloud. These are followed in the gravitational field of the SMBH, taking into account the hydrodynamical interaction with the diluted ambient wind. Gravitational effects need to be taken into account, but nonetheless, the Newtonian description is adequate for the gravitational field because we considered the motion with the pericentre at $\simeq 10^{3} r_{\mathrm{s}}$, so not in the immediate vicinity of the black hole horizon, where the relativistic effects on the orbit evolution become important. The variety of factors listed above underline a potentially important influence of the grain properties for the gas/dust coupling and hence support our simulation approach.

We employed the integration package Swift (Levison \& Duncan 1994), which has been designed to evolve a set of mutually gravitationally interacting bodies together with a group of test particles that are influenced by gravity of the massive bodies, but do not affect each other (or the massive bodies). In the numerical scheme we included the effect of radial wind outflow and that of ambient gaseous wind and employed a sufficiently accurate Bulirsch-Stoer integrator with an adjustable time-step.

The adopted procedure allowed us to follow a large number of numerical particles in the gravitational field, namely, a superposed gravitational field of the central SMBH and the embedded star. The gravity of the black hole plays a role in the motion of parcels because their mass is relatively large (like dust grains compared to gas atoms), but we modified the integration routine to also include the hydrodynamic drag that acts through the interaction of particles with the gaseous ISM as well as an outflowing stellar wind. The dust grain mass is a parameter that allowed us to study different cases, including a toy model where the grains represent super-particles of mass exceeding that of realistic cosmic dust.

\subsection{Non-gravitational forces acting on dust}

Although the stellar atmosphere consists largely of outflowing gas, here we concentrated mainly on the dust component. Both species interact with each other, but they can separate to a certain extent during the evolution. Observationally, the gas is expected to be revealed by $\mathrm{Br} \gamma$ spectral line emission, while the dust grains will contribute to the thermal continuum and can be detected by polarisation. This provides information about in situ conditions of the surrounding environment, but the predictions are currently uncertain due to incomplete knowledge about the nature and composition of G2/DSO. 
In a galactic nucleus all objects interact with the hot plasma that is supplied by stellar winds, in particular, of hot, massive OB stars. The effects of this interaction depend on temperature and density profiles of the ISM. For the purpose of this work, we used semi-analytical relations for electron density $n_{\mathrm{e}}(r)$ and electron temperature $T_{\mathrm{e}}(r)$ radial profiles based on the models of radiatively inefficient accretion flows (Broderick \& Loeb 2006; Broderick et al. 2011),

$n_{\mathrm{e}}(r)=n_{\mathrm{e}}^{0}\left(\frac{r}{r_{\mathrm{s}}}\right)^{-1.1}, \quad T_{\mathrm{e}}(r)=T_{\mathrm{e}}^{0}\left(\frac{r}{r_{\mathrm{s}}}\right)^{-0.84}$.

In these dependencies, we considered quantities $n_{\mathrm{e}}$ and $T_{\mathrm{e}}$ to adopt averaged values, $n_{\mathrm{e}}^{0}=3.5 \times 10^{7} \mathrm{~cm}^{-3}$ and $T_{\mathrm{e}}^{0}=9.5 \times$ $10^{10} \mathrm{~K}$. Electrons are decoupled from ions at small distances from the $\mathrm{SMBH}$; ion temperatures are $\sim 1$ to 5 times higher than electron temperatures resulting from MHD simulations (e.g., Dexter et al. 2010). The density profile (2) is used in Eq. (7) to estimate the ambient density $\rho_{\mathrm{a}}=m_{\mathrm{H}} n_{\mathrm{H}}$ (with $\left.n_{\mathrm{H}} \approx n_{\mathrm{e}}\right)$.

The profiles in eq. (2) were originally derived for the accretion flows up to $\sim 100 r_{\mathrm{s}}$, whereas we considered the pericentre passages at a typical distance one order of magnitude larger, $r \sim 10^{3} r_{\mathrm{s}}$. However, the densities inferred from the onedimensional model (used to fit Chandra X-ray data farther away from the Galactic centre; Quataert 2004) do not differ much when extrapolated from the inner flow to the region of our interest (factor of 3; see Psaltis 2012, for comparisons).

In general, the equation describing the motion of dust particles in the gaseous environment (optically thin stellar atmospheres, protoplanetary discs) is

$\frac{\mathrm{d} v}{\mathrm{~d} t}=\boldsymbol{a}_{\mathrm{grav}}+\boldsymbol{a}_{\mathrm{drag}}+\boldsymbol{a}_{\mathrm{rad}}$

where $\boldsymbol{a}_{\text {grav }}$ represents gravitational interactions, $\boldsymbol{a}_{\text {drag }}$ stands for the wind drag acceleration, and $\boldsymbol{a}_{\mathrm{rad}}$ is the acceleration due to radiation pressure.

The radiation pressure acceleration acting on a grain that is located at distance $r_{\star}$ from the star with flux $\Phi_{\star}=L_{\star} /\left(4 \pi r_{\star}^{2}\right)$ may be expressed by (e.g., Bertotti et al. 2003)

$\boldsymbol{a}_{\mathrm{rad}}=Q \frac{\Phi_{\star} \sigma_{\mathrm{eff}}}{m c}\left[\left(1-\frac{\boldsymbol{r}_{\star} \cdot \boldsymbol{v}_{\star}}{r_{\star} c}\right) \frac{\boldsymbol{r}_{\star}}{r_{\star}}-\frac{\boldsymbol{v}_{\star}}{c}\right]+O\left(\frac{v_{\star}^{2}}{c^{2}}\right)$,

where the factor $Q$ reflects the way the particle absorbs or reflects light, $m$ denotes the dust mass, $\sigma_{\text {eff }}$ its effective crosssection, $\boldsymbol{v}_{\star}$ is the velocity vector in the frame of the star, and $c$ is the speed of light. The terms in the brackets represent the direct pressure, the change of radiation energy due to the Doppler effect, and the Poynting-Robertson drag, respectively. Equation (4) is valid in a Newtonian approximation that is consistent with the pericentre passage of a star at the distance of $\sim 1000 r_{\mathrm{s}}$ at most. However, in the immediate vicinity of the SMBH, it would be necessary to include the second and higher powers of $\left(v_{\star} / c\right)$ as well.

The first two radial terms in Eq. (4) effectively change the mass of the star, which itself is a free parameter in our model, and the non-radial Poynting-Robertson term causes the decrease in the semimajor axis and eccentricity of the orbit and contributes to the inspiral of dust towards the star on the PoyntingRobertson time scale $\tau_{\mathrm{pr}}$, which may be approximated using
Eq. (4) and setting the effective cross-section equal to the geometrical cross-section of the grain, $\sigma_{\text {eff }}=\pi R^{2}$ :

$$
\begin{aligned}
\tau_{\mathrm{pr}} & =\frac{16 \pi c^{2}}{3 Q} L_{\star}^{-1} R \rho_{\mathrm{d}} r^{2} \\
& =28 \times 10^{2} \mathrm{yr} \frac{1}{Q}\left(\frac{L_{\star}}{L_{\odot}}\right)^{-1}\left(\frac{R}{1 \mu \mathrm{m}}\right)\left(\frac{\rho_{\mathrm{d}}}{1 \mathrm{~g} \mathrm{~cm}^{-3}}\right)\left(\frac{r}{1 \mathrm{AU}}\right)^{2} .
\end{aligned}
$$

In our model, we worked with time scales of $100 \mathrm{yr}$, which are shorter than $\tau_{\mathrm{pr}}$ by one order of magnitude for distances of $\sim 1$ AU. For more distant orbits, the time scale is significantly prolonged because of its quadratic dependence on the distance.

The radiation pressure from other S-stars in the background also contributes, but it is at least two orders of magnitude weaker. For an estimate, we took $\sim 500$ mas $\approx 4125 \mathrm{AU}$ as an average distance of individual S-cluster members. The estimated number of $\sim 20-M_{\odot}$ stars is $\sim 30$ and we compared the effect of their radiation with a low-mass (LM) star of $2 M_{\odot}$. Hence, the ratio of accelerations due to radiation, Eq. (4), is

$$
\begin{aligned}
\frac{a_{\mathrm{LM}}}{a_{\mathrm{S}}} & \approx \frac{L_{\mathrm{LM}}}{30 L_{\mathrm{S}}}\left(\frac{r_{\mathrm{S}}}{r_{\mathrm{LM}}}\right)^{2} \\
& \approx \frac{1}{30}\left(\frac{M_{\mathrm{LM}}}{M_{\mathrm{S}}}\right)^{3.5}\left(\frac{r_{\mathrm{S}}}{r_{\mathrm{LM}}}\right)^{2} \\
& =\frac{1}{30}\left(\frac{2 M_{\odot}}{20 M_{\odot}}\right)^{3.5}\left(\frac{4125 \mathrm{AU}}{1 \mathrm{AU}}\right)^{2} \approx 180 .
\end{aligned}
$$

Similarly, the Poynting-Robertson time scale, Eq. (5), yields $\tau_{\mathrm{pr}} \approx 10^{5} \mathrm{yr}$ for the average distance of 500 mas and the luminosity of $\sim 3020-M_{\odot}$ stars. Scoville \& Burkert (2013) also estimated that although a production rate of Lyman continuum photons is high, very few of them are intercepted because of the small area of the bow shock formed ahead of the star. Hence, the effect of radiation pressure from background stars is weak on the time scale of $\sim 100 \mathrm{yr}$ considered here.

The radiation drag in the vicinity of a putative star will only slightly affect individual orbits of grains on the time scale of a few orbital periods. The ensemble of particles behaves similarly with and without the radiation term involved, which shows that gravitational forces and hydrodynamical drag are dominant for their dynamics. To reduce computation costs, we omitted this term in most of our numerical calculations.

The acceleration caused by the drag for both supersonic (Stokes law) and subsonic (Epstein law) modes may be written in a closed form (e.g., Kwok 1975; van Marle et al. 2011):

$\boldsymbol{a}_{\text {drag }}=-\eta_{\text {drag }} \frac{\pi R^{2}}{m} \rho_{\mathrm{a}} \sqrt{v_{\text {rel }}^{2}+\bar{v}_{\mathrm{t}}^{2}} \boldsymbol{v}_{\text {rel }}$,

where $\boldsymbol{v}_{\text {rel }}=\boldsymbol{v}-\boldsymbol{v}_{\mathrm{g}}$ is the relative velocity of grains with respect to the gas motion $\boldsymbol{v}_{\mathrm{g}}$. The mean thermal velocity, assuming Maxwellian distribution, is $\bar{v}_{\mathrm{t}}=(8 / \pi)^{1 / 2} c_{\mathrm{s}}$, which is proportional to the sound speed $c_{\mathrm{s}}=\sqrt{k T / m_{\mathrm{H}}}$ (for ideal gas). The factor $\eta_{\text {drag }}$ acquires the value $4 / 3$ for the Epstein law and $1 / 2 C_{\mathrm{d}}(R e)$ for the Stokes law, where the coefficient $C_{\mathrm{d}}(R e)$ depends on the Reynolds number $R e$ (for an in-depth discussion see Perets \& Murray-Clay, 2011). As shown in Fig. 2, orbital velocities within the inner $\sim 10^{6}$ Schwarzschild radii are supersonic, $v_{\text {orb }} \approx \sqrt{G M_{\bullet} / r}>c_{\mathrm{s}}$, so the Stokes law is approximately valid. This is also true for grains embedded in the outflowing wind atmosphere close to the star, see Fig. 3. If we assume an isothermal atmosphere with $T \approx 10000 \mathrm{~K}$ that is in ionisation equilibrium with the environment, orbital velocities are approximately 
Michal Zajaček et al.: Dust-enshrouded star near supermassive black hole

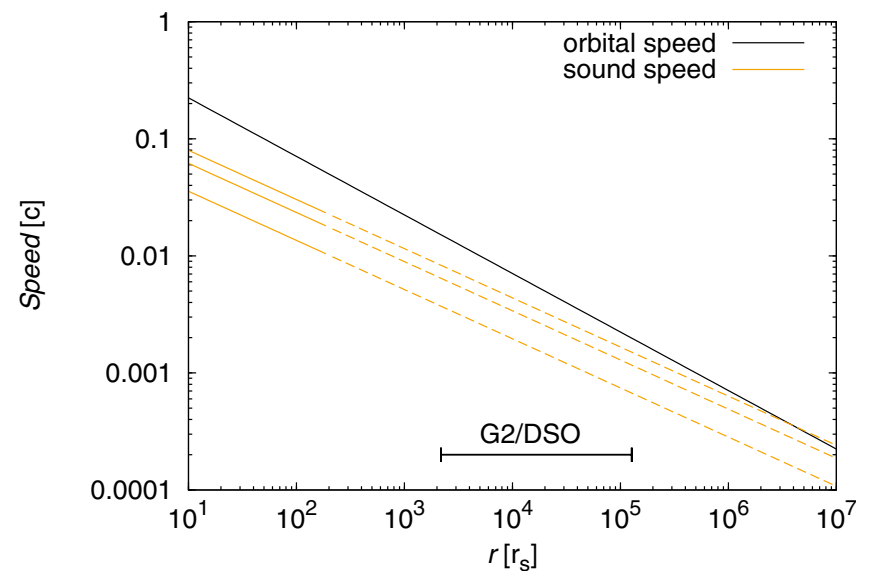

Fig. 2. Comparing the orbital (Keplerian) velocity with the sound speed at corresponding radius from the central black hole (units of Schwarzschild radii). The range of G2/DSO is labelled. On the vertical axis, the unit of speed is the speed of light, $c$.

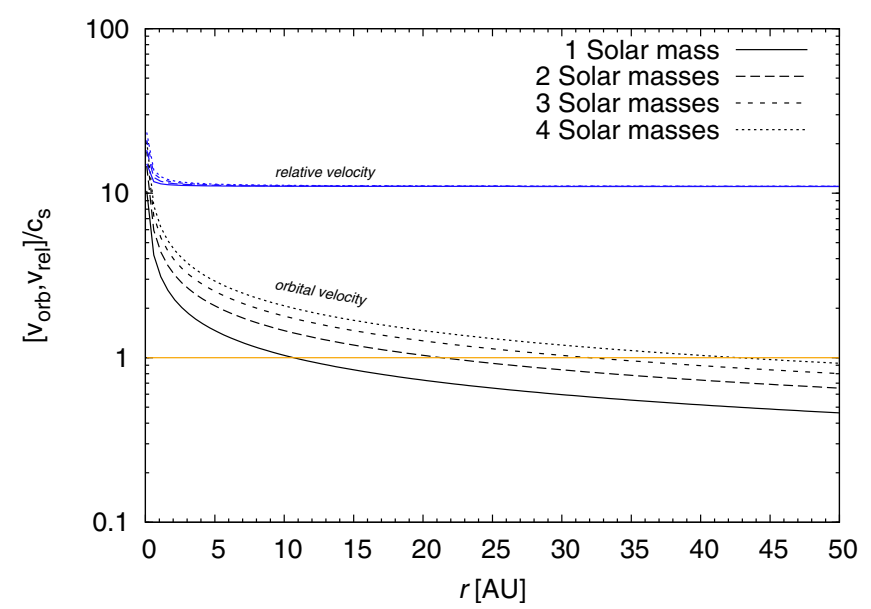

Fig. 3. Ratio of orbital velocities (black lines) and relative velocities (blue lines) to the speed of sound as a function of distance from the star. Relative velocities are typically higher than the sound speed by about one order of magnitude for wind velocities of the order of $100 \mathrm{~km} \mathrm{~s}^{-1}$.

equal to the sound speed at distance $r_{\mathrm{s}} \approx G M_{\star} m_{\mathrm{H}} /(k T)$ and decrease. However, relative velocities with respect to radial outflow, in case of circular orbits of grains, are higher than the sound speed by about factor of 10 for typical terminal wind velocities of $100 \mathrm{~km} \mathrm{~s}^{-1}$. Assuming that grains have a spherical shape with diameter $d$ and substituting the dust grain volume times density for the mass, we obtain $\boldsymbol{a} \approx-\frac{3}{4} C_{\mathrm{d}}(R e)\left(\rho_{\mathrm{a}} / \rho_{\mathrm{d}}\right) d^{-1} v_{\text {rel }} \boldsymbol{v}_{\text {rel }}$ and we set $C_{\mathrm{d}}=1^{2}$.

We note that the magnetic field frozen in the stellar wind and potentially that of the interstellar medium near the galactic nucleus as well may influence the motion of charged dust, mainly of a smaller size. This is mainly determined by the ratio of the charge of the grain $q$ resulting from photo-charging and its mass $m, q / m$. When the main component of the field is radial, $\boldsymbol{B}=B_{r} \boldsymbol{r}$, and dust motion is azimuthal, $\boldsymbol{v}=v_{\phi} \hat{\boldsymbol{\phi}}$, the Lorentz acceleration, $a_{\mathrm{L}}=(q / m) v_{\phi} B_{r}$ causes the grain inclination to increase or decrease, depending on the mutual orientations of the magnetic field and velocity vectors. The field orientation may flip after some time and may lead to oscillations around the orbital plane. Because of the many uncertainties concerning the

\footnotetext{
2 From subsonic to supersonic regimes, the drag coefficient changes typically by about a factor of two.
}
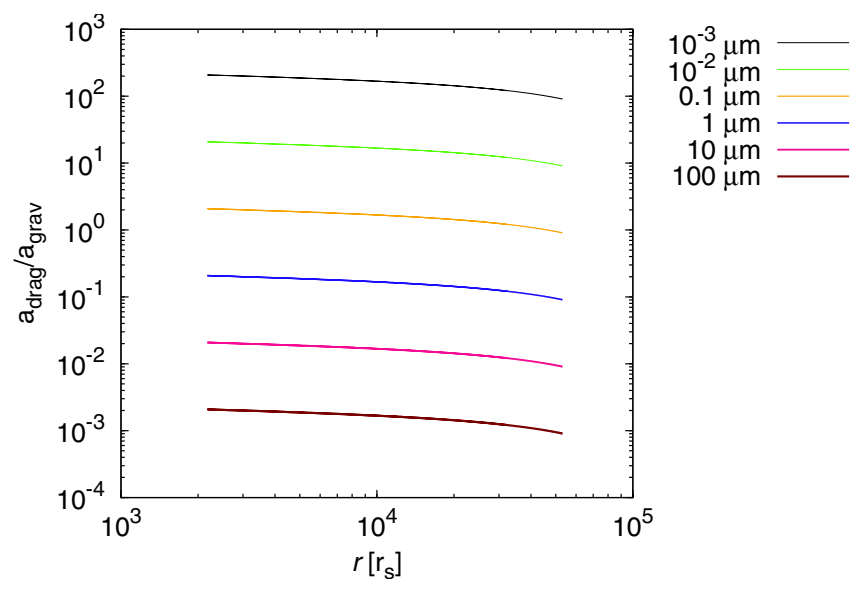

Fig. 4. Ratio of the hydrodynamical drag to the gravitational acceleration across the range of radii. The two effects can be of comparable magnitude and, therefore, both need to be taken into account in the model of the orbit evolution.

magnetic field strength and the efficiency of photo-charging, we did not consider the magnetic field in our analysis.

Despite the arguments for neglecting these terms and effects, this is just a crude model. Nonetheless, it allows us to reproduce the relevant trends in a semi-analytical way.

In Fig. 4 we plot the ratio of the magnitude of the acceleration due to the drag force (7) with respect to the gravitational acceleration across the range of radii. In this case we neglected the motion of ambient medium and set the relative velocity $v_{\text {rel }}$ equal to the orbital speed. Although the ratio varies significantly (by six orders of magnitude), it is non-negligible for some typical particle sizes in the range $\sim 0.1 \mu \mathrm{m}-1 \mu \mathrm{m}$ (for the smallest particles it exceeds the gravitational acceleration magnitude $)^{3}$.

As the cloud approaches the central region, the dust absorbs the radiation mainly at shorter wavelengths and heats up. The dust temperature grows to the sublimation temperature $T_{\text {sub }}$ and eventually leads to sublimation. When we take into account only the radiation of the central accretion flow, the sublimation radius is easily determined (e.g., Barvainis 1987):

$r_{\mathrm{sub}}=567 T_{\mathrm{sub}}^{-2.8}\left[\left(\frac{L_{\mathrm{uv}}}{L_{\odot}}\right) \exp \left(-\tau_{\mathrm{uv}}\right)\right]^{\frac{1}{2}}[\mathrm{pc}]$,

where $L_{\mathrm{uv}}$ stands for the luminosity of the central source at ultraviolet wavelengths expressed in units of solar luminosity $L_{\odot}$. The optical depth, $\tau_{\mathrm{uv}}$, enables us to estimate the sublimation radius or inversely the temperature of dust at given $r$ (again, realistic profiles must be more complicated; Kishimoto et al. 2011). Furthermore, in sources with radiatively efficient accretion, X-ray heating contributes significantly (Czerny \& Hryniewicz 2011).

For the sublimation temperature $T_{\text {sub }} \approx 1500 \mathrm{~K}$ (Barvainis 1987), low optical depth, and $L_{\mathrm{uv}} \approx 10^{3} L_{\odot}$, the relation (8) gives $r_{\mathrm{sub}} \approx 2.3 \times 10^{-5} \mathrm{pc} \approx 56 r_{\mathrm{s}}$ as a lower estimate (we did not consider hot $\mathrm{OB}$ stars present near the centre, whose radiation probably heats the dust further). When the stellar populations are taken into account, the central luminosity can be estimated to a few $\sim 10^{6} L_{\odot}$ for the smooth distribution, and up to $10^{7} L_{\odot}$ for the

3 The effect might be more significant in AGN, where the typical density of the environment is higher than in inactive nuclei, but in that case strong irradiation leads to dust heating and destruction in a relatively large volume around the central source. 


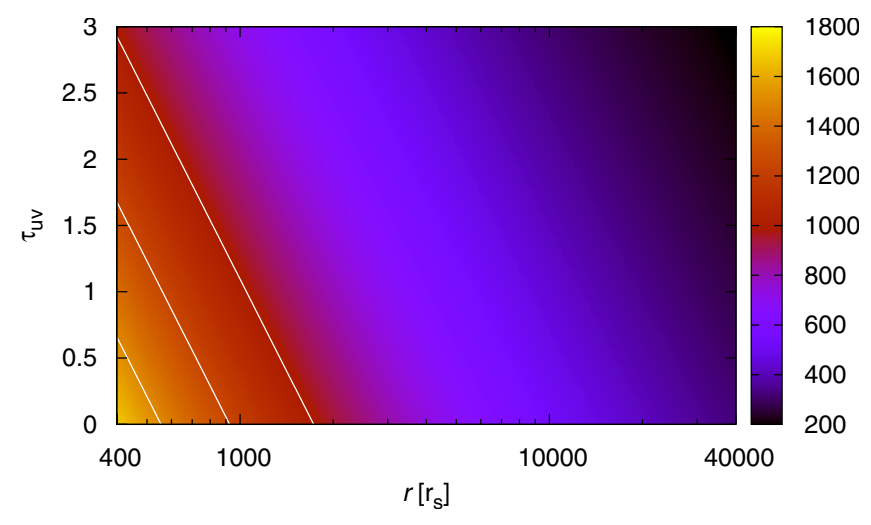

Fig. 5. Colour-coded profile of temperature $T \equiv T\left(r, \tau_{\mathrm{uv}}\right)$ of dust for the central source of luminosity $10^{5} L_{\odot}$ near the central SMBH. From right to left, the three lines are isotherms corresponding to $T=1000 \mathrm{~K}$, $1250 \mathrm{~K}$, and $1500 \mathrm{~K}$ (dust sublimation temperature).

clumpy structure (Davidson et al. 1992), which leads to $r_{\text {sub }} \approx$ $2.3 \times 10^{-3} \mathrm{pc} \approx 5600 r_{\mathrm{s}}$ as an upper limit for the sublimation radius.

Given the uncertainty, it is appropriate to refer to the sublimation zone extending from a few tens of Schwarzschild radii up to $\sim 5 \times 10^{3} r_{\mathrm{s}}$. To shorten the integration time of numerical experiments, we safely set the inner sublimation radius to $300 r_{\mathrm{s}}$ in our test runs. Using the inverse relation $T=T\left(r, \tau_{u v}\right)$ in Eq. (8), we estimate the temperature profile of dust near the source of central luminosity $10^{5} L_{\odot}$, which is intermediate between the limits of $10^{3}$ and $10^{7} L_{\odot}$ (see Fig. 5).

The passages with pericentre above $\simeq 1 \times 10^{3} r_{\mathrm{s}}$ (the case of G2/DSO, the Dusty S-cluster Object in the Galactic centre $)^{4}$ probably does not heat the dust to the sublimation temperature, which would only be reached at $\approx 600 r_{\mathrm{s}}$. However, the mechanism of heating is more complicated not only because of the presence of stars, but also because of the shock heating that may contribute in case of transonic motion.

\subsection{Role of central star}

It has often been advocated (e.g., Gillessen et al. 2013b) that the infalling DSO/G2 source may represent a core-less clump that has originated farther out at greater distance, $\gtrsim 10^{4} r_{\mathrm{s}}$, and now is on the way toward the pericentre, where it will largely disintegrate and undergo accretion onto the SMBH. However, it may be also a dusty envelope that enshrouds a star. Extended shells surrounding stars near Sgr A* have been reported, see for example Moultaka et al. (2005).

Before the pericentre passage, the two cases are expected to produce a similar image and the corresponding spectrum to contain a thermal component from the dust. On the other hand, a transit through the pericentre must reveal the nature in a clearer way. Because we considered a pericentre distance of the order of $10^{3} r_{\mathrm{s}}$, the star itself is not destroyed by tidal forces of the SMBH, instead, it continues to follow the eccentric ellipse. The diluted atmosphere is more visibly affected by its interaction with the ambient medium.

First, we compared the temporal evolution of the spatial distance and velocity with/without the hydrodynamical drag for the high-eccentricity orbits of a single particle. As a specific

\footnotetext{
4 The S-cluster is the $1^{\prime \prime}$ diameter star cluster of high-velocity stars surrounding Sgr A* (Eckart \& Genzel 1996; Ghez et al. 1998; Eckart et al. 2005; Melia 2007; Genzel et al. 2010).
}

example, we used the $L^{\prime}$-band based nominal orbital elements of G2/DSO (Gillessen et al. 2013a). The resulting evolution is plotted in Fig. 6; it appears to be consistent with eccentricities and semi-major axes of dust grains gradually decreasing, as they move in the ambient medium.

Next, we considered a cloud of particles with a certain initial distribution of positions and velocities and included different effects acting on the motion. Gillessen et al. (2012, 2013a) studied the evolution of such a cloud of particles in the field of the Sgr A* SMBH. We revisited their simulations by including the interaction with different prescriptions for the ambient wind. In our simulations, for example, 2000 particles were initially distributed according to a spherically symmetric Gaussian distribution in the position-velocity phase space. We used the same $L^{\prime}$-band-based orbital elements for the G2/DSO cloud as above.

The evolution in the orbital plane and in the velocity-distance plane are plotted in Fig. 7 (left panels) for selected epochs since the start of the simulation. The initial FWHM of the phase-space distribution was set to be 25 mas for positions (the value adopted from Gillessen et al. 2013b), and $5 \mathrm{~km} \mathrm{~s}^{-1}$ as a typical turbulent velocity within the cloud. These simulations reveal that because of the drag of the ambient ISM and gravity of the SMBH, most particles continue to spiral in towards the centre. The cloud as a whole becomes progressively stretched by the tidal forces, and the velocity dispersion rises abruptly as particles approach the SMBH.

Secondly, we examined the evolution with an embedded star, whose mass has been set to $M_{\star}=2 M_{\odot}$. Dust grains $(d=5 \mu \mathrm{m})$ initially obey a uniform distribution of semi-major axes in the range $(0.1,50) \mathrm{AU}$, eccentricities in the range $(0,0.1)$, and inclinations within $\left(0^{\circ}, 180^{\circ}\right)^{5}$. The distribution of velocities is Keplerian. Figure 7 also shows the evolution of such a stellar source (right panels).

The particles start to trail behind the star because of the drag. In combination with the tidal effects, the position and the velocity dispersion gradually increase up to the pericentre. During the post-pericentre phase the velocity dispersion first decreases, then increases again while particles spiral inwards to the SMBH. In Fig. 7 we clearly see how the attraction of the star influences some of the particles (cp. the epoch 15.05). A small fraction of particles remain bound to the star; specifically for this run, about $5 \%$ particles remained bound to the star; this number did not change after the second pericentre passage.

\subsection{Dust truncation radius}

To estimate the dust mass diverted from the initial nominal trajectory of the cloud at its pericentre passages, it is useful to consider the Hill radius of the binary system star-SMBH. At the pericentre,

$r_{\mathrm{H}}=a(1-e)\left(\frac{M_{\star}}{3 M_{\bullet}}\right)^{1 / 3}$,

which for the mass $M_{*}=2 M_{\odot}$ and the position at the pericentre yields $\sim 1 \mathrm{AU}$; inside this sphere of influence the particles remain bound to the star, while outside it the particles are captured by the $\mathrm{SMBH}$.

The sphere of influence increases after each pericentre passage, as illustrated in Fig. 8, where we plot the Hill radius evolution over an interval of time. We varied the eccentricity, but

5 Inclination values lower than $90^{\circ}$ correspond to a prograde (direct) orbit, values exceeding $90^{\circ}$ are reserved for the retrograde sense of motion. 
Michal Zajaček et al.: Dust-enshrouded star near supermassive black hole
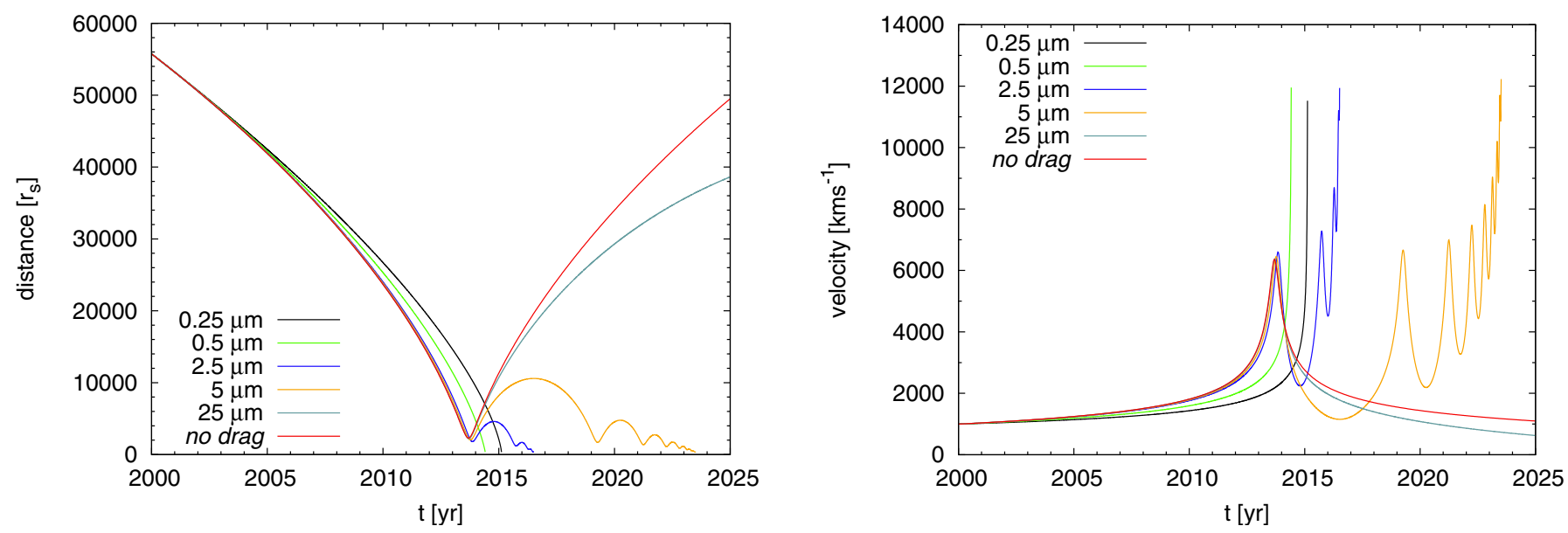

Fig. 6. Single-particle approximation. Left panel: the temporal evolution of 3D distance (in Schwarzschild radii); the orbital parameters are set consistent with the G2/DSO in Galactic centre. Different particle (dust grain) sizes are considered, taking into account the effect of gravity and the hydrodynamical drag exerted by the ambient medium. Right panel: the corresponding velocity as a function of time. Hydrodynamical drag acts more efficiently on particles of larger size, causing their rapid in-spiralling and accretion onto the central black hole.
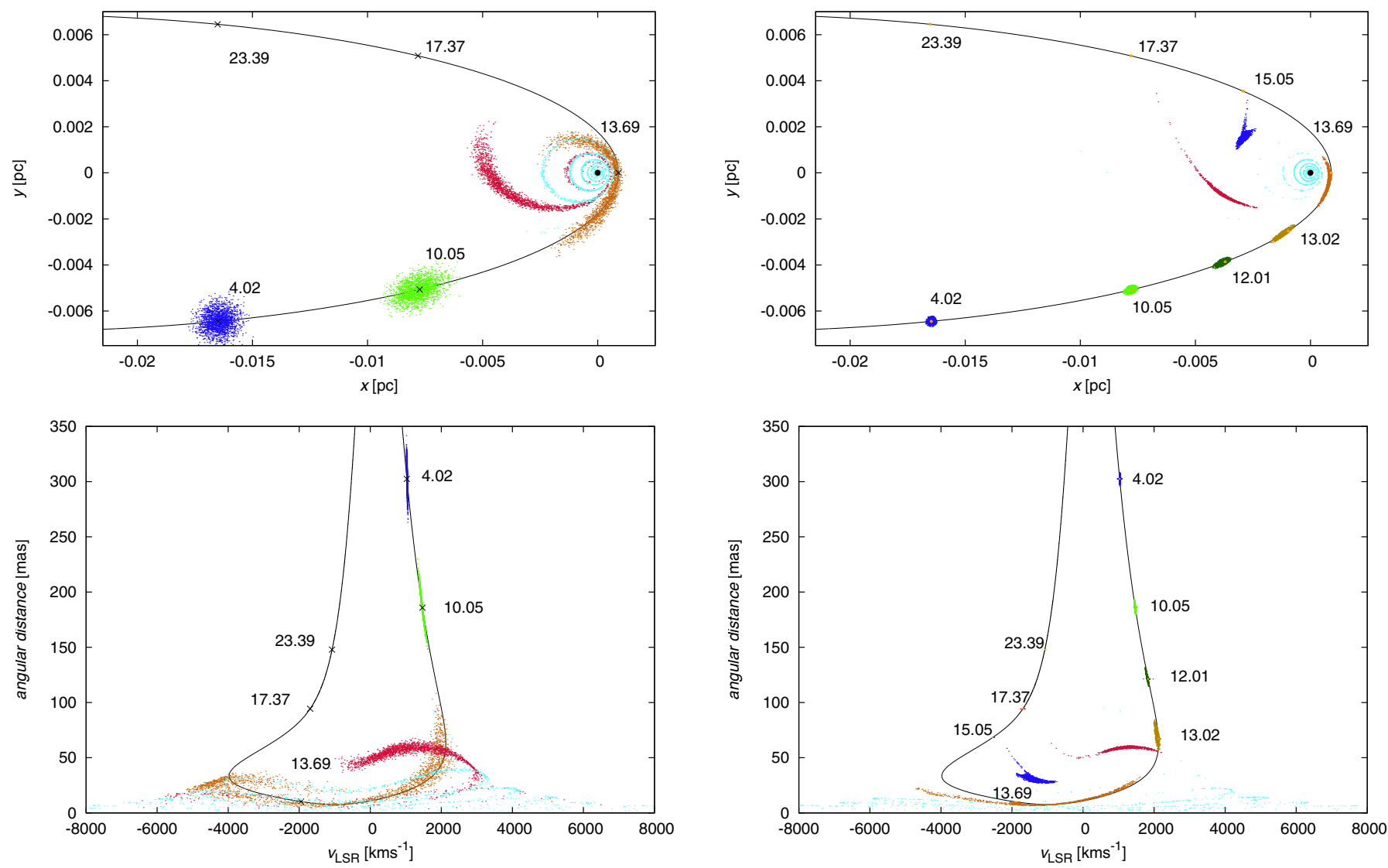

Fig. 7. Numerical representation of the dust/gas cloud in terms of elementary parcels, trajectories of which are integrated in the gravitational field and influenced by the hydrodynamical drag. Top left: the evolution of the cloud in the orbital plane for selected epochs since the start of the simulation. Snapshots of the cloud are distinguished by different colours and shown at several moments of time (time marks correspond to years since the initial moment of integration). The cloud particles are characterised by the Gaussian dispersion around the nominal trajectory in the phase space (the black curve). Bottom left: representation of the evolution from the top-left panel is shown in the velocity-distance plane. The velocity is transformed into the local standard of rest. The angular distance is expressed in miliarcseconds. Top right: the evolution as in the top-left panel, but for a cloud with an embedded star in the core. Bottom right: the track in the velocity-position plane corresponding to the trajectory from the top-right panel.

other orbital parameters remained fixed $\left(M_{\bullet}=4.4 \times 10^{6} M_{\odot}\right.$ for Sgr A* SMBH). Highly eccentric trajectories $(e \gtrsim 0.9)$ are prone to a significant mass loss when the atmosphere of the star undergoes the Roche-lobe overflow onto the black hole.
The Hill radius expressed by Eq. (9) employs only tidal shearing. However, in our case the motion of dust is influenced by drag from the wind outflow, and this may indeed decrease the critical radius at which orbits of grains become unstable and 


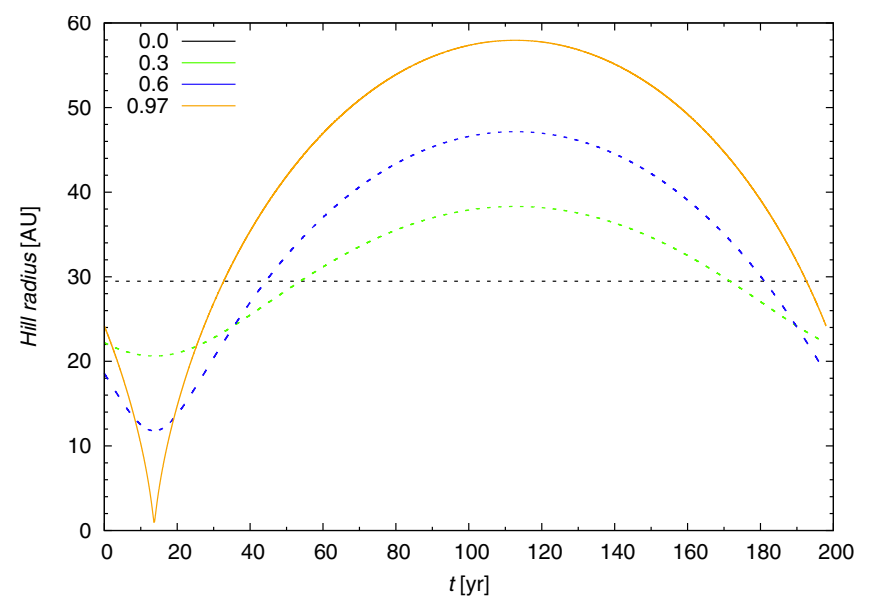

Fig. 8. Critical Hill radius $r_{\mathrm{H}}(t)$ for four different eccentricities: $e=0.0$, $0.3,0.6$, and 0.97. Enhanced accretion occurs when some of the dust particles move beyond $r_{\mathrm{H}}$.

consequently leave the circumstellar environment. Following the analysis of Perets \& Murray-Clay (2011), we define the windtruncation radius at which the gravitational acceleration acting on the grain $G M_{\star} / r_{\star}^{2}$ is equal to the acceleration $a_{\text {drag }}$ resulting from the wind drag:

$r_{\mathrm{w}}=\left(\frac{G M_{\star}}{a_{\mathrm{drag}}}\right)^{1 / 2}$.

After inserting the drag term (7) into (10), we obtain

$r_{\mathrm{w}}=\left(\frac{G M_{\star} m}{\eta_{\mathrm{drag}} \pi R^{2} \rho_{\star}\left(v_{\mathrm{rel}}^{2}+\bar{v}_{\mathrm{t}}^{2}\right)^{1 / 2} v_{\mathrm{rel}}}\right)^{1 / 2}$.

To derive an estimate, we assumed the Stokes law and rewrote Eq. (11) as a function of grain diameter $d$ and distance from the star $r_{\star}$,

$r_{\mathrm{w}}\left(d, r_{\star}\right)=\left(\frac{4}{3} \frac{G M_{\star}}{C_{\mathrm{d}}} \rho_{\mathrm{d}}\right)^{1 / 2}\left(\frac{d}{\rho_{\star} v_{\mathrm{rel}}^{2}}\right)^{1 / 2}$,

where both the stellar wind density $\rho_{\star}$ and the relative velocity of grains with respect to the wind $v_{\text {rel }}$ are functions of the distance from the star. We rewrote Eq. (12) into a convenient form, taking $\rho_{\mathrm{d}}=2260 \mathrm{~kg} \mathrm{~m}^{-3}$ for the density of dust (e.g., Barvainis 1987) and assuming the density of spherical wind outflow $\rho_{\star}=\dot{m}_{\mathrm{w}} /\left(4 \pi r_{\star}^{2} v_{\mathrm{w}}\right)$,

$$
\begin{aligned}
r_{\mathrm{w}}= & 0.28\left(\frac{M_{\star}}{M_{\odot}}\right)^{1 / 2}\left(\frac{d}{\mu \mathrm{m}}\right)^{1 / 2}\left(\frac{v_{\mathrm{rel}}}{100 \mathrm{~km} \mathrm{~s}^{-1}}\right)^{-1}\left(\frac{v_{\mathrm{w}}}{100 \mathrm{~km} \mathrm{~s}^{-1}}\right)^{1 / 2} \\
& \times\left(\frac{\dot{m}_{\star}}{10^{-8} M_{\odot} \mathrm{yr}^{-1}}\right)^{-1 / 2}\left(\frac{r_{\star}}{\mathrm{AU}}\right) \mathrm{AU} .
\end{aligned}
$$

In Fig. 9, we plot the wind-truncation radius as a function of both the grain diameter (in $\mu \mathrm{m}$ ) and the distance from $2 M_{\odot^{-}}$ star (in AU) with the typical mass-loss rate of $10^{-8} M_{\odot} \mathrm{yr}^{-1}$ and the terminal wind speed of $100 \mathrm{~km} \mathrm{~s}^{-1}$. The wind drag generally causes the stability region to shrink below the Hill radius (in our case $\sim 1 \mathrm{AU}$ at the pericentre), mainly affecting small grains $\lesssim 1 \mu \mathrm{m}$.

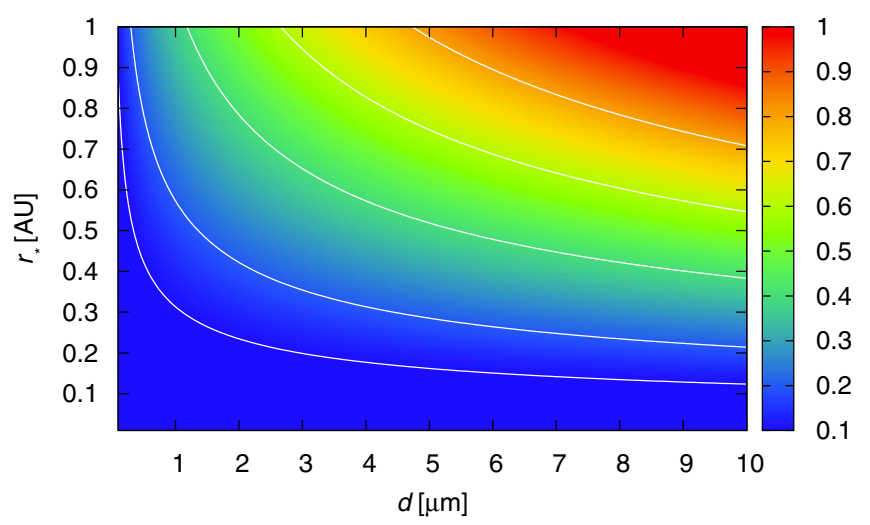

Fig. 9. Colour-coded plot of the wind-truncation radius (colour bar units in $\mathrm{AU}$ ). Each point in the graph estimates the truncation radius from the equilibrium between gravitational and drag forces for a given distance from the star and a grain diameter. The contours stand for values 0.1 , $0.2,0.4,0.6$, and $0.8 \mathrm{AU}$.

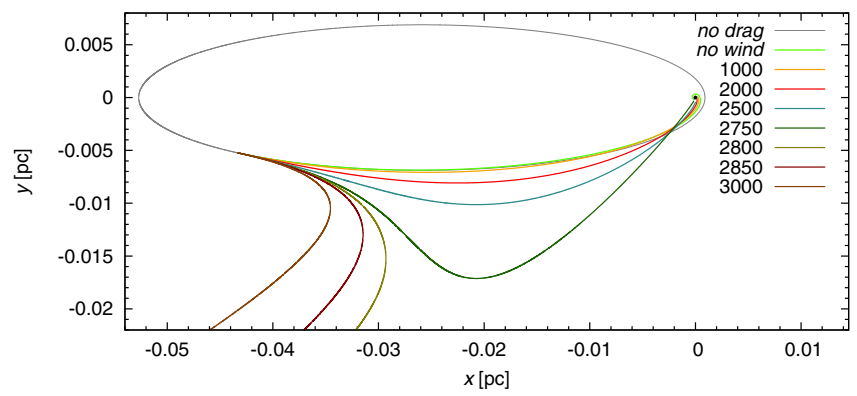

Fig. 10. Trajectory of a single dust grain $(d=1 \mu \mathrm{m})$ for different cases of the central wind outflow velocity. The grey ellipse corresponds to the nominal trajectory, neglecting the drag by the wind. The values of the outflow velocity are expressed in $\mathrm{km} \mathrm{s}^{-1}$ in the legend.

\section{Results of simulations}

\subsection{Effects of wind and a bow shock - the case of G2/DSO}

Comet-shaped features have been observed near the Galactic centre that point to the role of the radial outflow in the form of a wind from the central region (Mužić et al. 2010). The source of the wind can be hot stars and/or the accretion flow in the vicinity of the SMBH. A similar effect is expected to occur in our model as well. One of the differences that distinguishes the core-less cloud from a dust-enshrouded star is the size of the bow shock (e.g., van Marle et al. 2011; Araudo et al. 2013). In the case of a star, the bow shock is formed by the wind interaction; a powerful wind outflow (as in the case of massive stars) develops the stagnation point radius at a much larger radius than the size of the star. On the other hand, in the case of a core-less cloud the bow shock is only slightly larger than the size of the cloud.

We included the radial wind outflow by setting $\boldsymbol{v}_{\text {rel }}=\boldsymbol{v}-\boldsymbol{v}_{\mathrm{w}}$ in Eq. (7), where $v$ stands for the velocity of numerical particles with respect to the SMBH, and $\boldsymbol{v}_{\mathrm{w}}$ is the wind velocity (we assumed a spherical outflow at $r \lesssim 1^{\prime \prime}$ ). The particles feel the wind drag; the computed trajectories for particles of $d=1 \mu \mathrm{m}$ are shown in Fig. 10. The initial conditions were the same for all cases and were computed according to the nominal orbital elements of the G2/DSO object. For small and moderate wind velocities $\left(\lesssim 1000 \mathrm{~km} \mathrm{~s}^{-1}\right)$, the trajectory is only slightly affected. The time of pericentre passage is delayed and the rate of spiralling inwards increases because there is an additional 
Michal Zajaček et al.: Dust-enshrouded star near supermassive black hole
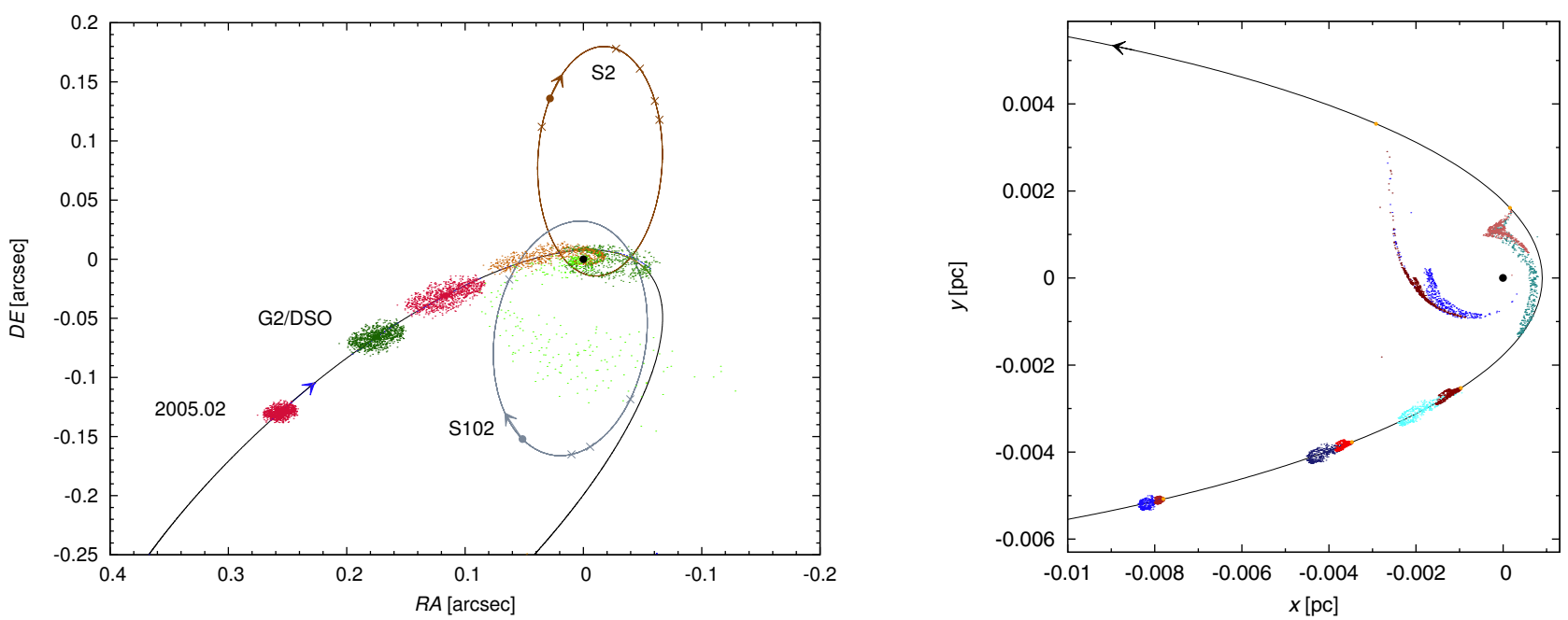

Fig. 11. Left panel: a sketch of the orbit orientation on the sky, consistent with the current nominal trajectory of the G2/DSO (i.e., arriving from the south-east direction and going into pericentre from the north, then leaving towards the south-east, showing the offset from the SMBH position in the origin). Two eccentric orbits of the S-stars are also indicated. Right panel: two cases of the possible evolution a gaseous/dusty stellar envelope, initially bound to the star: (i) with a radial wind blowing from the centre $\left(v_{\mathrm{w}}=1000 \mathrm{~km} \mathrm{~s}^{-1}\right.$; bluish colours); and (ii) without the outflowing wind (reddish colours). Here the same nominal trajectory as in the left panel is plotted, now with respect to the $(x, y)$ orbital plane. See the text for details.

non-radial term (due to the aberration). For $v_{\mathrm{w}} \gtrsim 2800 \mathrm{~km} \mathrm{~s}^{-1}$ the particles are blown away with the wind. Mužić et al. (2010) found evidence of the presence of wind speed from the centre that are of this order of magnitude.

Consequently, we compared the evolution of a bound stargas-dust system with and without the effect of a spherical wind from the centre. Particles $(d=2.5 \mu \mathrm{m})$ were initially distributed uniformly around the $\operatorname{star}\left(M_{\star}=3 M_{\odot}\right.$ in this example). The range of inclination was set from $0^{\circ}$ to $180^{\circ}$, semi-major axes spanned the interval $(0.001,10) \mathrm{AU}$, and eccentricities varied from 0 to 0.1 . The resulting evolution is plotted in Fig. 11. The wind slows the particles down and the initial shape of the shell is more stretched than in the no-wind case.

If a wind-blowing star moves supersonically through ISM, a bow shock is formed. The effect has also been confirmed at 0.2 pc distance from the Galactic centre (Mužić et al. 2010). Here we employed a two-dimensional model of the bow shock,

$R(\theta)=R_{0} \csc \theta \sqrt{3(1-\theta \cot \theta)}$,

which we rotated around the symmetry axis to obtain the correct orientation of the shock in $3 \mathrm{D}$. In Eq. (14), $R_{0}$ stands for the stand-off distance, where the ram pressures of the ambient medium and the stellar wind are at balance (Wilkin 1996),

$R_{0}=\left(\frac{\dot{m}_{\mathrm{w}} v_{\mathrm{w}}^{\star}}{4 \pi \rho_{\mathrm{a}} v_{\mathrm{rel}}^{2}}\right)^{1 / 2}$,

where $\dot{m}_{\mathrm{w}}$ is the stellar mass-loss rate, $v_{\mathrm{w}}^{\star}$ is the terminal velocity of the stellar wind. We used Eq. (2) to find $\rho_{\mathrm{a}}$, and $v_{\text {rel }}=\left|\boldsymbol{v}-\boldsymbol{v}_{\mathrm{w}}\right|$ at a specific point on the orbit. The symmetry axis of the bow shock is aligned with the direction of the relative velocity, $\boldsymbol{v}_{\text {rel }}$, as expected.

We assumed that the wind-blowing star has a spherical wind with the constant magnitude equal to its terminal velocity. In our numerical scheme, particles outside the bow shock feel the drag and wind from the ambient medium near the nucleus. Inside the bow shock, the drag is approximately proportional to the second power of the relative velocity with respect to the circumstellar environment with the radial outflow, and to the circumstellar density $\rho_{\star}, \rho_{\star}=\dot{m}_{\mathrm{w}} /\left(4 \pi r_{\star}^{2} v_{\mathrm{w}}^{\star}\right)$. In further examples we used the parameters relevant for a young, low-mass (T Tauri) star (Scoville \& Burkert 2013): $\dot{m}_{\mathrm{w}}=10^{-8} M_{\odot} \mathrm{yr}^{-1}$, $v_{\mathrm{w}}^{\star}=200 \mathrm{~km} \mathrm{~s}^{-1}$, but the procedure may be also applied to more evolved stellar types.

Now we also included the effect of the bow shock on the cloud evolution. In our example we set the mass of an embedded star to $M_{\star}=2 M_{\odot}$ and compared two cases (Fig. 12): first, the wind blowing from the centre at velocity of $500 \mathrm{~km} \mathrm{~s}^{-1}$, and, second, the other case with $1000 \mathrm{~km} \mathrm{~s}^{-1}$ wind. In each, case particles have a uniform distribution of semi-major axes in the interval $(0.1,50) \mathrm{AU}$, inclination $\left(0^{\circ}, 180^{\circ}\right)$, and eccentricity $(0,0.1)$. We note that the character and orientation of the bow-shock sources can in principle be tested observationally via polarimetry (Buchholz et al. 2013; Valencia-S et al. 2012), although we did not attempt this here.

The evolution is similar in the two cases - the material outside the bow shock spirals towards the SMBH because of the drag, while inside the bow shock the cloud elements continue to move through the circumstellar environment. Figure 12 shows a very narrow shape of the bow shock near the pericentre. At this stage, many particles are torn away from the star, which is enhanced by both stellar wind and the outflow from the centre (see Sect. 2.4). However, a fraction that occupies the bow-shock region continues to orbit. In the case of strong wind from the centre, the particles are dragged more efficiently, so that a long tail forms behind the star. As mentioned above, only a diminishing part of the initial material survives the repeating passages through pericentre. The cloud is progressively stretched and accreted, and its centre drifts away from the position of the embedded star.

We also plot the changing division between the predicted position of the star and the centre of mass of the cloud (Fig. 12, bottom row). The mutual separation tends to grow with time (apart from fluctuations) till the pericentre passage, then decreases again as the fraction of the cloud unbound to the star sublimates or becomes accreted onto the black hole. This offset is expected to be detectable in the NIR band if sufficient resolution is reached to resolve the cloud structure. 

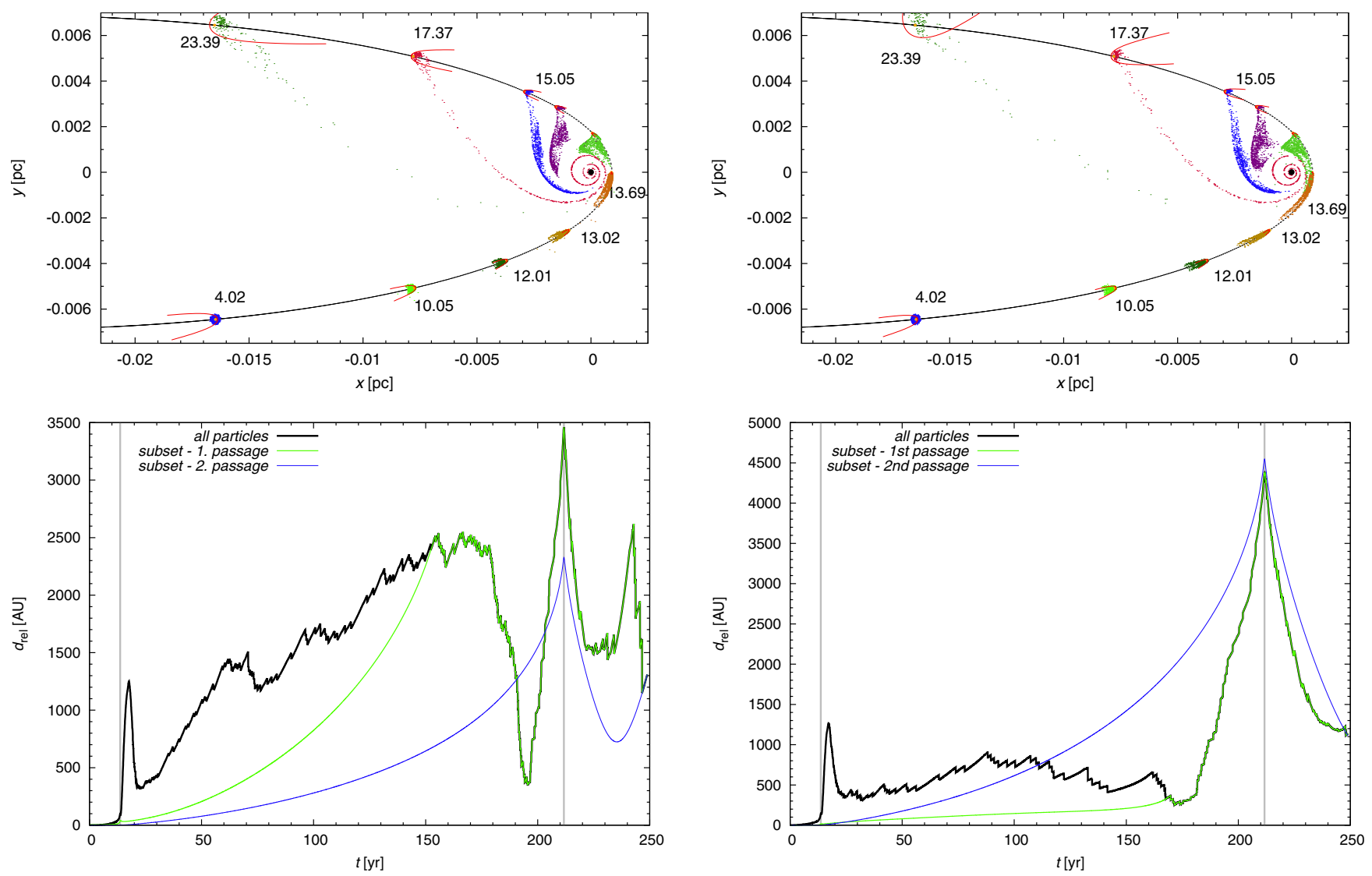

Fig. 12. Evolution of the star with a dusty envelope together with an instantaneous orientation of the bow shock (top panels). Top left: star mass $M_{\star}=2 M_{\odot}$. The velocity of the wind from the centre is $v_{\mathrm{w}}=500 \mathrm{~km} \mathrm{~s}^{-1}$. Top right: same parameters as in the left panel except for $v_{\mathrm{w}}=1000 \mathrm{~km} \mathrm{~s}^{-1}$, showing the change of orientation of the bow shock. In both cases, the mass transfer peaks with a slight delay after the pericentre passage. Corresponding to the two panels in the top row, an offset is shown between the star position along its nominal orbit from the centre of mass of the cloud in the bottom panels. Different curves show the offset computed for all particles forming the cloud (black curve), a subset of particles in the cloud that survive the first passage through the pericentre (green curve), and a subset of those that also survive the second passage. Moments of pericentre passages are indicated by vertical lines. The separation of 1000 AU corresponds to $\simeq 0.12$ arcsec at the distance to Sgr A*. Bottom left: $v_{\mathrm{w}}=500 \mathrm{~km} \mathrm{~s}^{-1}$. Bottom right: $v_{\mathrm{w}}=1000 \mathrm{~km} \mathrm{~s}^{-1}$.

\subsection{Spherical cloud vs. disc-like structure}

Near a protostar or T Tauri star, two types of dust/gas environment can be considered: a spherical cloud with an approximately Gaussian distribution in the position-velocity phase space, and a disc-like configuration with a Keplerian distribution of bulk velocity. The latter case represents a protoplanetary or a debris disc. Murray-Clay \& Loeb (2012) proposed such a disc as the origin of the cloud near SMBH. We compared the evolution of the two geometries.

In both cases, dust grains are distributed around a $2 M_{\odot}$ star with the parameters from the previous section. For the Gaussian velocity dispersion cloud, the initial FWHM was taken to be equal to $100 \mathrm{AU}(\approx 12 \mathrm{mas})$ and $5 \mathrm{~km} \mathrm{~s}^{-1}$, respectively. Particles in the disc were distributed uniformly, with semi-major axes in the range $(0.1,50) \mathrm{AU}$, low eccentricity in the range $(0,0.1)$, and inclinations in the range $(0,30)^{\circ}$ and $(150,180)^{\circ}$ (taking into account both direct and retrograde orbits with respect to the orbit of the star).

In Fig. 13 we plot a typical evolution in the orbital plane for the chosen epochs (top panels) as well as the distribution in the position-velocity diagrams (bottom panels). These simulations indicate that a Gaussian cloud is not as much affected by the star as the particles in the disc. It is also evident that in case of the disc structure, more particles survive the pericentre passage and continue to orbit the star.

\subsection{Fraction of mass influenced at subsequent encounters}

High-eccentricity passages are more likely to be non-repeating events if the cloud does not host a body inside its volume (see Fig. 7). The material of the cloud becomes dispersed and largely accreted onto the SMBH at the first pericentre passage. However, in the scenario with a shell surrounding a stellar object, a fraction of material remains bound to the star while only the rest is rerouted towards the black hole. In Table 1, we summarise the typical results of computations of the percentage of the captured material for the first and the subsequent pericentre passages.

As soon as the star/cloud on its trajectory reaches the critical radius for the mass overflow, the percentage of captured mass is high ( $\gtrsim 90 \%$ at the first pericentre passage). For the Gaussian distribution in the position-velocity phase space, it is near or equal to $100 \%$ in both the bow-shock and no-bow-shock cases. When the bow shock is included and particles follow the bulk Keplerian distribution in a disc, more particles remain bound after the first pericentre crossing than the no-bow-shock scheme predicts (the bow-shock region protects some particles from being diverted from the cloud orbit near the pericentre). This is connected with the character of the drag forces on both sides of the bow shock in our model and also agrees with previous results concerning the fate of a pressure supported gas cloud, which appears to be particularly prone to complete destruction near the pericentre. However, one should bear in mind that we work with 
Michal Zajaček et al.: Dust-enshrouded star near supermassive black hole
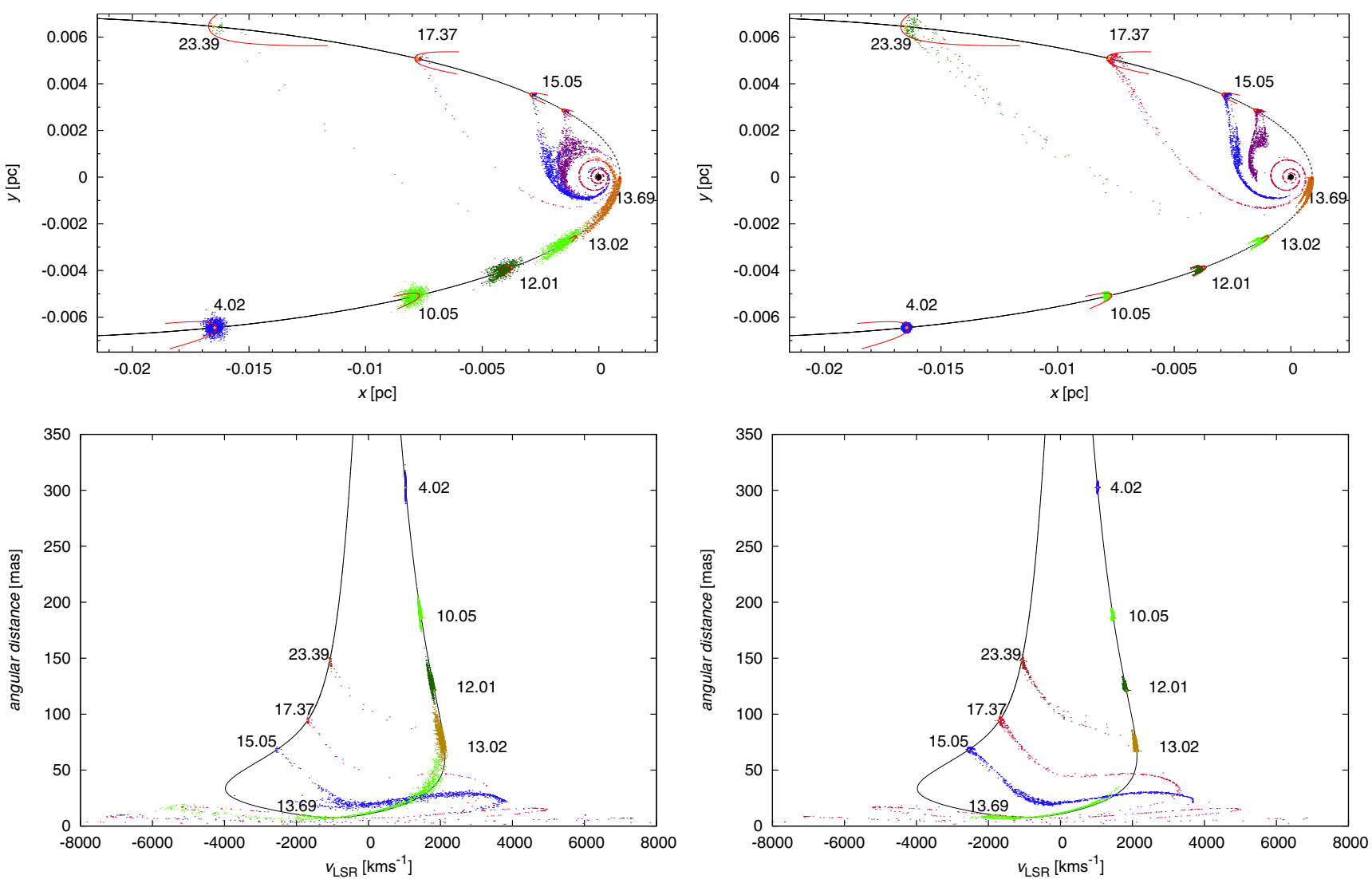

Fig. 13. Analogy to Fig. 7 for the case with the bow-shock. Top left: the evolution of an initially spherical Gaussian envelope in the presence of a star for chosen epochs since the start of the simulation. Bottom left: representation of the evolution from the top-left panel is shown in the velocity-distance plane. The angular distance is expressed in miliarcseconds. Top right: as in the top-left panel, but for a star-disc system. Bottom right: the track in the velocity-position plane corresponds to the trajectory from the top-right panel.

Table 1. Typical examples from different runs for the fraction of material deflected from the original trajectory during the first and second pericentre passages (evaluated with respect to the total number of particles in the cloud at the corresponding stage).

\begin{tabular}{|c|c|c|c|c|c|c|c|}
\hline \multirow[t]{2}{*}{ Run } & \multicolumn{4}{|c|}{ Model characteristics } & \multirow[t]{2}{*}{ Note } & \multicolumn{2}{|c|}{ Mass capture [\%] } \\
\hline & Initial distribution & Bow shock & $M_{\star} / M_{\odot}$ & Grain size $[\mu \mathrm{m}]$ & & 1st passage & 2nd passage \\
\hline 1 & disc-like (dr) & no & 3 & 2.5 & - & 99.2 & 0.0 \\
\hline 2 & spherical & no & 3 & 2.5 & - & 100.0 & 0.0 \\
\hline 3 & disc-like (dr) & no & 3 & 2.5 & - & 99.4 & 0.0 \\
\hline 4 & disc-like (dr) & no & 3 & 5 & - & 99.6 & 0.0 \\
\hline 5 & disc-like (dr) & no & 2 & 5 & - & 99.6 & 50.0 \\
\hline 6 & disc-like (dr) & no & 2 & 5 & - & 99.5 & 0.0 \\
\hline 7 & disc-like (dr) & no & 3 & 2.5 & (i) & 95.2 & 12.5 \\
\hline 8 & disc-like (dr) & no & 3 & 2.5 & (i), (ii) $1000 \mathrm{~km} \mathrm{~s}^{-1}$ & 94.4 & 14.3 \\
\hline 9 & disc-like (dr) & yes & 2 & 2.5 & (ii) $500 \mathrm{~km} \mathrm{~s}^{-1}$ & 86.6 & 83.6 \\
\hline 10 & disc-like (dr) & yes & 2 & 2.5 & (ii) $1000 \mathrm{~km} \mathrm{~s}^{-1}$ & 88.6 & 58.8 \\
\hline 11 & spherical & yes & 2 & 2.5 & (ii) $500 \mathrm{~km} \mathrm{~s}^{-1}$ & 99.2 & 100.0 \\
\hline 12 & disc-like (dr) & yes & 2 & 2.5 & (ii) $500 \mathrm{~km} \mathrm{~s}^{-1}$ & 91.3 & 65.5 \\
\hline 13 & disc-like (dr) & yes & 2 & 0.6 & (ii) $500 \mathrm{~km} \mathrm{~s}^{-1}$ & 91.3 & 71.3 \\
\hline 14 & disc-like (dr) & yes & 2 & 0.1 & (ii) $500 \mathrm{~km} \mathrm{~s}^{-1}$ & 98.1 & 100.0 \\
\hline 15 & disc-like (dr) & yes & 2 & 2.5 & (ii) $500 \mathrm{~km} \mathrm{~s}^{-1}$ & 87.3 & 90.6 \\
\hline 16 & sphere+disc (dr) & yes & 3 & 2.5 & (ii), $1000 \mathrm{~km} \mathrm{~s}^{-1}$, rad & 93.3 & 68.7 \\
\hline 17 & disc-like (d) & yes & 2 & 2.5 & (ii), $1000 \mathrm{~km} \mathrm{~s}^{-1}$, rad & 88.6 & 74.8 \\
\hline 18 & disc-like (r) & yes & 2 & 2.5 & (ii), $1000 \mathrm{~km} \mathrm{~s}^{-1}$, rad & 90.6 & 89.8 \\
\hline
\end{tabular}

Notes. Columns of model characteristics specify the initial distribution of particle positions and velocities (disc-like Keplerian vs. spherical Gaussian), presence or absence of the bow-shock effect and wind, assumed mass of the star in the cloud core, and size of the dust grains. (i) dust region extending from $r=0.001 \mathrm{AU}$; (ii) central outflow wind $v_{\mathrm{w}}$ included; "rad" denotes radiation pressure from the star; (d, r, dr) denotes only direct orbits, only retrograde orbits, and both, respectively. Additional (non-essential) parameter is the number of numerical particles in each run (typically of the order of $10^{3}$ ). 

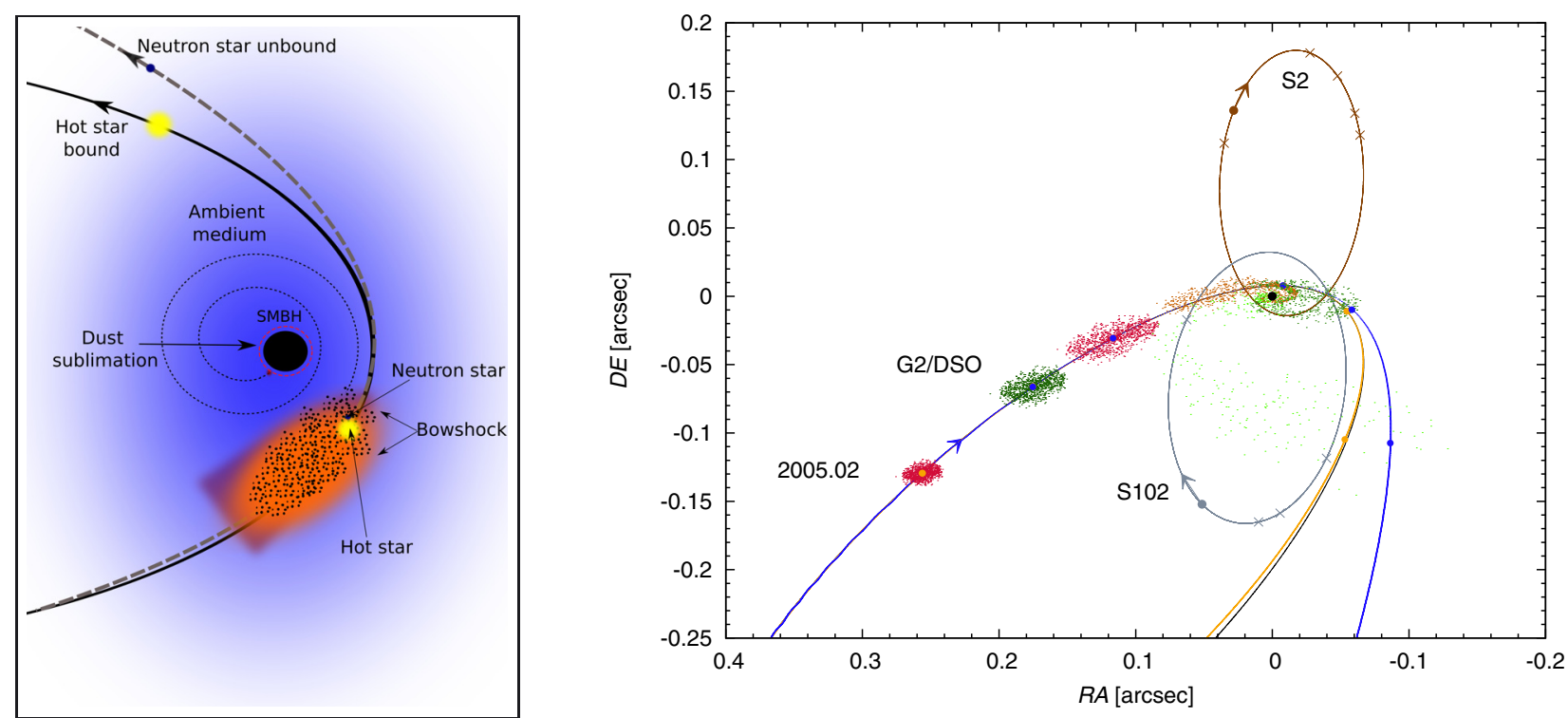

Fig. 14. Sketch of the model geometry for a binary with a common envelope (in the left panel) and the orientation consistent with the G2/DSO nominal trajectory on the sky (in the right panel). The two components (i.e., hot star+neutron star in this example) deviate from each other, the first one being bound and the other one unbound at the moment of pericentre passage. The cloud is then dispersed in the ambient medium and eventually accreted onto the SMBH.

a four-parametric model. At the second pericentre passage, the total amount of the captured material is generally much smaller than at the first passage, nevertheless, the percentage fluctuates significantly in terms of the immediate mass of the cloud.

We also tested a potential difference between the stability of direct (prograde) and retrograde discs. From the classical threebody theory, one can derive the difference between the critical Hill radii of direct and retrograde orbits, $r_{\mathrm{d}}$ and $r_{\mathrm{r}}$, respectively, resulting from the Coriolis term (Innanen 1979):

$\frac{r_{\mathrm{r}}}{r_{\mathrm{d}}}=\left[\frac{5+e+2(4+e)^{1 / 2}}{3+e}\right]^{2 / 3}$,

which leads to the ratio of $r_{\mathrm{r}} / r_{\mathrm{p}} \approx 1.9$ for the eccentricity $e \approx 1$, which is our case. Hence, retrograde discs may not be as truncated as direct discs; the upper limit to the prolongation of their critical radius is the factor of $\sim 1.9$. This is consistent with our test runs involving only gravity. A direct disc around a $2-M_{\odot}$ star with the semi-major axes in the range $(0.05,10) \mathrm{AU}$, low inclinations $(0,10)^{\circ}$, and low eccentricities $(0,0.01)$ dissolves after one revolution after the first pericentre passage, forming families of orbits along the original trajectory and trajectories with smaller semi-major axes, with some particles escaping the system. A few of them remain in orbit of the star inside the Hill radius. A retrograde disc with the same distribution of orbital elements remains more compact and detached particles spread mostly along the original trajectory. However, when drag forces from both the stellar wind and the central outflow are involved, there is no difference in terms of stability between direct and retrograde discs (see runs 17 and 18 in Table 1). This is because the stability of discs is no longer determined by the gravitational Hill radius, but by the smaller wind-truncation radius (see Sect. 2.4).

These results demonstrate that in the most cases the stellar envelope is significantly affected around the pericentre, and the diverted part eventually becomes largely dispersed during the accretion event. The outcome is similar to the case of a gaseous cloud, treated in the hydrodynamical regime (Anninos et al. 2012; Burkert et al. 2012), although a small fraction of the dusty envelope can survive to the following revolution.

\subsection{Binary embedded within common envelope}

The high eccentricity of the G2/DSO trajectory suggests that it might be possible to connect this object with hypothetical events of the three-body interaction (Hills 1988) involving the SMBH as the origin of stars on bound orbits near SMBH (Gould \& Quillen 2003, see Fig. 14). In this tentative scenario a binary system is disrupted during the pericentre passage. As a result of this interaction, one of the remnant bodies remains on a circularised orbit, whereas the other component gains orbital energy and is ejected (Löckmann et al. 2008; Bromley et al. 2012). For realistic estimates of disruption rates, see Perets et al. (2007).

Might the infalling cloud contain such a binary stellar system? The initial eccentric trajectory of the binary centre of mass was set in agreement with the infalling cloud within which it remain embedded. The orbital parameters of the binary system are expected to be disturbed at the moment of close encounter with the SMBH and the observational resolution about the nature of the object should emerge soon after the pericentre passage. We can illustrate two qualitatively different options for the possible outcome of the post-pericentre evolution: (i) both components remain bound to the SMBH; (ii) one component is ejected from the system on a hyperbolic trajectory at the expense of the orbital energy of the other component.

For each case we performed a number of simulations with a different setup of free parameters, namely, the masses $M_{\star}^{(i)}$ of the two components and the osculating elements of the embedded binary system. The primary component $M_{\star}^{(1)}$ represents a hot, wind-blowing star, the secondary $M_{\star}^{(2)}$ is thought to be a neutron star (for definiteness of the simulation). The gaseousdusty envelope enshrouding the whole binary was modelled in the same way as in previous examples, that is, in terms of dust particles that experience the hot atmosphere of the SMBH, the stellar wind from the primary and the bow-shock effect. The initial conditions and parameters are summarised in Table 2. In general, the fraction of cloud initial mass that is retained after the pericentre passage is diminished by the presence of the binary star in the core compared with an otherwise similar set-up with a single stellar object embedded inside. 
Table 2. Two exemplary cases of an embedded binary evolution.

\begin{tabular}{l|cc}
\hline \hline Parameter & Case A & Case B \\
\hline Mass of the binary components $\left[M_{\odot}\right]$ & 4.0 & 3.0 \\
& 1.4 & 1.4 \\
True anomaly [ $\left.{ }^{\circ}\right]$ & 120.0 & 80.0 \\
Semi-major axis $[\mathrm{AU}]$ & 3.0 & 2.0 \\
Eccentricity & 0.05 & 0.05 \\
$\dot{m}_{\mathrm{w}}\left[M_{\odot} \mathrm{yr}^{-1}\right]$ & \multicolumn{2}{|c}{$10^{-7}$} \\
$v_{\mathrm{c}}\left[\mathrm{km} \mathrm{s}^{-1}\right]$ & \multicolumn{2}{|c}{500} \\
$v_{\mathrm{w}}^{\star}\left[\mathrm{km} \mathrm{s}^{-1}\right]$ & \multicolumn{2}{|c}{700} \\
\hline
\end{tabular}

Notes. The true anomaly is the initial value, $\dot{m}_{\mathrm{W}}$ stands for the mass-loss rate. Additional wind parameters $v_{\mathrm{c}}$ and $v_{\mathrm{w}}^{\star}$ denote the terminal velocities of the assumed spherical wind from the centre and the star, respectively. In these examples we performed integration runs with $1000 \mathrm{nu}-$ merical particles representing the material of an initially spherical cloud, whereas the circumbinary disc population consisted of 500 particles with semi-major axes uniformly distributed from $10 \mathrm{AU}$ to $50 \mathrm{AU}$, inclinations up to $30^{\circ}$, and eccentricities ranging from 0.0 to 0.1 . The spherical cloud population adopts a Gaussian distribution in the phase space with the initial FWHMs of 12.5 mas and $5 \mathrm{~km} \mathrm{~s}^{-1}$. In both examples, the integration starts at the true anomaly of $-167^{\circ}$ for the barycentre position.

In Fig. 15 we show the distance-velocity plots, the temporal evolution of the binary semimajor axis $a$, and eccentricity $e$. At the pre-pericentre phase, the components orbit the common centre of mass, while at the pericentre they become unbound with respect to each other and start to move independently with different semi-major axes and eccentricities. We note, however, that this is a multi-parametric system where the outcome of the evolution depends on a mutual interplay of different parameters.

The disruption event occurs when the secondary is outside the tidal-shearing radius, which may be again estimated using the Hill radius of the primary, Eq. (9). For cases A and B, we derived $r_{\mathrm{H}} \sim 1.3 \mathrm{AU}$ and $\sim 1.2 \mathrm{AU}$, respectively. Following the discussion in the previous subsection, the disruption radius for the binary orbiting the black hole approximated by Eq. (9) is effectively enlarged if the secondary orbits the primary in a retrograde sense.

The observation of a single event of the binary disruption while passing close to the SMBH is improbable and should be regarded only as a speculative scenario. However, other proposed scenarios for the G2/DSO infrared source, such as isothermal clouds or the disruption of a star, are also rare given the rate at which streams and winds collide or stars disrupt to produce such a cloud. Moreover, the nature of the binary content in the Galactic centre is still unclear (Antonini \& Perets 2012). Hence, all the estimates of binary replenishment in the central region are crude. The binary scenario can be easily rejected/confirmed by observations based on the post-pericentre evolution of the trajectory of the G2/DSO infrared source.

\section{Discussion}

We examined the pericentre passages and gradual destruction of dusty envelopes of stars that move supersonically through the immediate vicinity of the SMBH. Motivation for this topic arises because stars are shown to interact with the ionised medium close to Sgr A*, forming bow-shock structures (e.g., Mužić et al. 2010, and references therein). Here we focused on stars with a significant dust content that interacts with an optically thin wind outflow. Examples of such stars may also be found in the disc population of the Galaxy, specifically Herbig Ae/Be-type stars, see for instance Boersma et al. (2009).

The potential importance of the subject is heightened by the current passage of the infrared-excess G2/DSO source near Sgr A*. We focused on stellar-origin scenarios in which a star is enshrouded by a dusty shell. We revisited a simplified core-less scenario for comparisons of time scales and position-velocity distributions, although we did not perform detailed hydrodynamical simulations. The adopted approach is in several aspects complementary to hydrodynamical and MHD schemes, and it appears relevant for exploration of the dust component. We computed the evolution in the presence of the star and the SMBH and also included additional effects within an approximation:

- hydrodynamical drag due to the plasma environment in the SMBH inner accretion zone;

- radially directed wind outflowing from the centre;

- wind-shearing in the immediate circumstellar environment;

- bow-shock formation due to supersonic motion through the ISM.

We plotted the results showing the shape of a Keplerian disclike system and a Gaussian envelope at different epochs. We computed the line-of-sight velocities (transformed to the local standard of rest) and the angular distance-velocity plots.

The idea of stars embedded in clouds of gas and dust does not have to be limited to our Galaxy. It has been explored by various authors (e.g., Zurek et al. 1994; Armitage et al. 1996; Šubr \& Karas 1999; Vilkoviskij \& Czerny 2002, and references therein) in the context of repetitive interactions of stars of the nuclear cluster with the material of the accretion disc or a dusty torus in AGN.

The mini-spiral of the Galactic centre is a potential source of matter for infalling clouds. This structure contains a mixture of hot, warm, and cold phases. Under such conditions the accreted medium consists of complex plasma with a nonnegligible content of dust (Chan et al. 1997). Located at a distance about $0.1-0.2 \mathrm{pc}$ (projected distance $\sim 0.06 \mathrm{pc}$ ) from the supermassive black hole, this feature can be understood as consisting of three independent clumpy streams of mainly gaseous material at roughly Keplerian motion around the centre (Zhao et al. 2010; Kunneriath et al. 2012). The streams collide, and their mutual interaction may cause the loss of angular momentum and an occasional inflow of the clumpy material towards the black hole (Paumard et al. 2005; Czerny et al. 2013a). In this scenario, the distribution of angular momentum determines the probability of setting the clouds on a plunging orbit (Czerny et al. 2013b).

One can thus expect that the current example of G2/DSO in the centre of the Milky Way may be a signature of a common mechanism that transports the gaseous clumpy medium and stars to the immediate vicinity of the central SMBH on sub-parsec scales.

\section{Conclusions}

We modelled the fate of an infalling star with an extended envelope near the SMBH. The complex medium was treated in terms of numerical particles with their mass and size as parameters, interacting with the ambient environment. The mass of dust particles is typically large enough so that the gravitational influence on the grains needs to be taken into account close to the SMBH and the mass-losing star. 

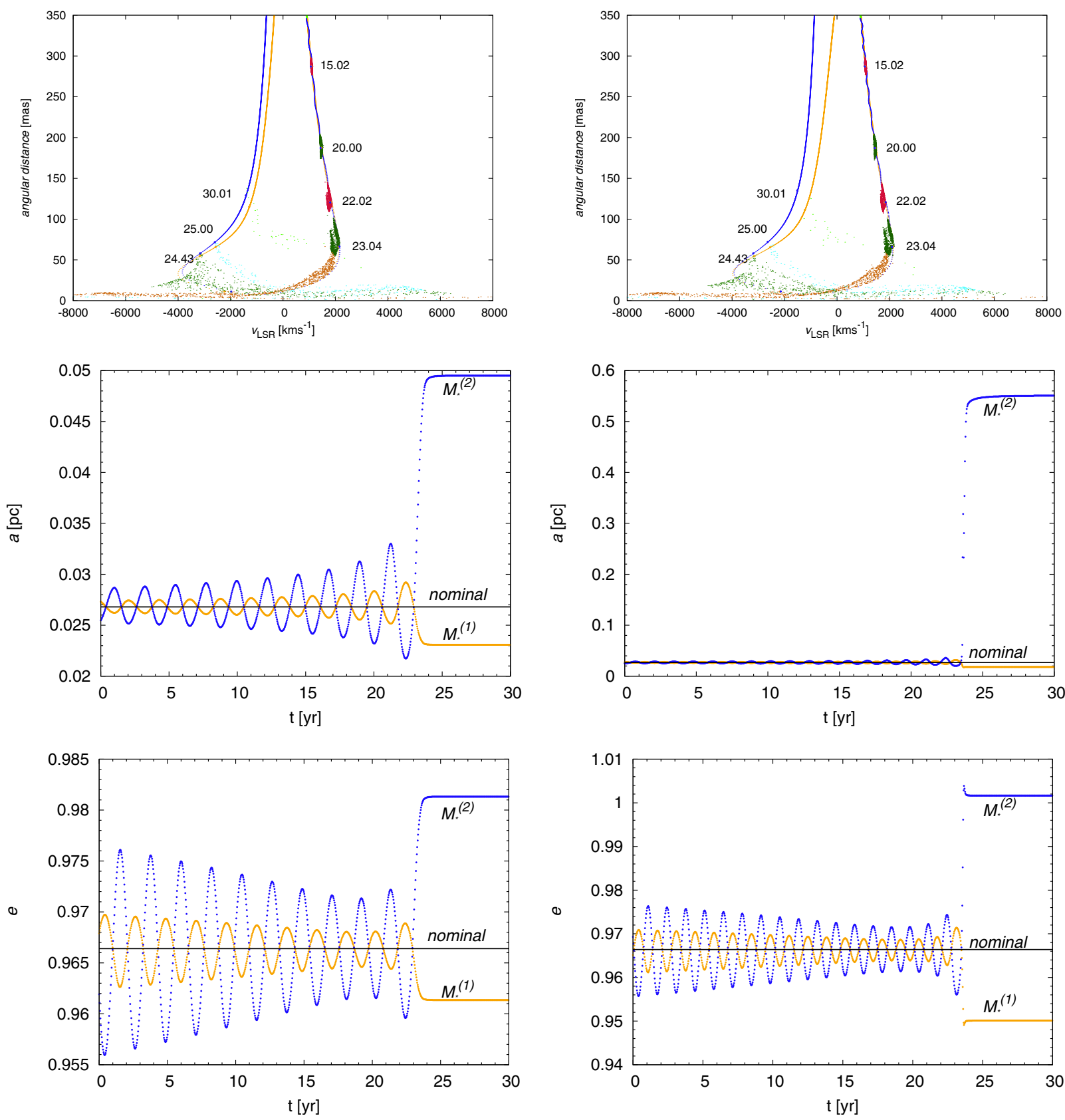

Fig. 15. Evolution of the cloud with an embedded binary, components of which become unbound via the three-body interaction at the pericentre passage near the central SMBH. The initial trajectory of the binary barycentre corresponds to the nominal trajectory of the G2/DSO (black solid line), whereas the post-pericentre stage depends strongly on the path of the individual components emerging from the system. Two exemplary cases are shown in different columns, corresponding to the choice of the component masses $M_{\star}^{(i)}$. Left panel: $M_{\star}^{(1)}=4.0 M_{\odot}, M_{\star}^{(2)}=1.4 M_{\odot}$. Right panel: $M_{\star}^{(1)}=3.0 M_{\odot}, M_{\star}^{(2)}=1.4 M_{\odot}$.

We assumed an orbit pericentre of the order of $10^{3} r_{\mathrm{s}}$, so that the star itself was not tidally disrupted. However, the surrounding cloud was affected very significantly (core-less clouds are influenced even more, and they are basically destroyed on the first encounter with the black hole). We noticed a significant mass-loss from the cloud at the first pericentre passage in all considered cases ( $\gtrsim 90 \%)$. During the second passage the mass loss fluctuates. In other words, if the star is enshrouded in a dusty shell before the first pericentre passage, it becomes stripped of most of the envelope, unless the material is continuously replenished. If a binary star is embedded within the envelope, there is a chance that the two components separate during the pericentre passage, revealing the nature of the cloud core.
During the pericentre passage, the centre of mass of the cloud separates from the stellar core inside the cloud, but then it returns to the star as the unbound particles are destroyed by sublimation or become accreted onto the black hole. The presence of the bow shock around a star somewhat diminishes the amount of captured particles (Table 1), and a greater fraction of the cloud can survive to the following pericentre passage. On the other hand, the presence of a binary tends to dissolve the cloud more efficiently at the moment of close encounter with the SMBH. The characteristics of motion across the bow shock depend strongly on parameters, mainly the mass-loss rate and the stellar-wind velocity, so the predictions are uncertain and the outcome of the simulations vary. In addition, there are still 
uncertainties in the density and temperature profiles of the flow near the Galactic centre. However, the comparison of our simulations with post-pericentre observations can help to set better constraints.

Acknowledgements. We thank Miroslav Brož for discussions and help with the Swift integration package. We are grateful to Hagai B. Perets and Ladislav Šubr for critical, constructive comments. The research leading to these results has received funding from the student grant of the Charles University in Prague (GAUK 879113), and the collaboration project of the Czech Science Foundation and Deutsche Forschungsgemeinschaft (GACR-DFG 13-00070J). The Astronomical Institute has been operated under the program RVO:67985815 in the Czech Republic.

\section{References}

Abramowicz, M. A., Czerny, B., Lasota, J. P., \& Szuszkiewicz, E. 1988, ApJ, 332,646

Anninos, P., Fragile, P. C., Wilson, J., \& Murray, S. D. 2012, ApJ, 759, 132

Antonini, F., \& Perets, H. B. 2012, ApJ, 757, 27

Antonucci, R. 1993, ARA\&A, 31, 473

Araudo, A. T., Bosch-Ramon, V., \& Romero, G. E. 2013, MNRAS, 436, 3626

Armitage, P. J., Zurek, W. H., \& Davies, M. B. 1996, ApJ, 470, 237

Ballone, A., Schartmann, M., Burkert, A., et al. 2013, ApJ, 776, 13

Barai, P., Proga, D., \& Nagamine, K. 2012, MNRAS, 424, 728

Barvainis, R. 1987, ApJ, 320, 537

Bertotti, B., Farinella, P., \& Vokrouhlicky, D. 2003, Physics of the Solar System - Dynamics and Evolution, Space Physics, and Spacetime Structure, Astrophys. Space Sci. Lib., 293,

Boersma, C., Peeters, E., Martín-Hernández, N. L., et al. 2009, A\&A, 502, 175 Broderick, A. E., \& Loeb, A. 2006, ApJ, 636, L109

Broderick, A. E., Fish, V. L., Doeleman, S. S., \& Loeb, A. 2011, ApJ, 738, 38 Bromley, B. C., Kenyon, S. J., Geller, M. J., \& Brown, W. R. 2012, ApJ, 749, L42

Buchholz, R. M., Witzel, G., Schödel, R., \& Eckart, A. 2013, A\&A, 557, A82

Burkert, A., Schartmann, M., Alig, C., et al. 2012, ApJ, 750, 58

Chan, K.-W., Moseley, S. H., Casey, S., et al. 1997, ApJ, 483, 798

Cox, D. P. 2005, ARA\&A, 43, 337

Czerny, B., \& Hryniewicz, K. 2011, A\&A, 525, L8

Czerny, B., Karas, V., Kunneriath, D., \& Das, T. K. 2013a, in IAU Symp., eds C. M. Zhang, T. Belloni, M. Méndez, \& S. N. Zhang (Cambridge: Cambridge University Press), 290, 199

Czerny, B., Kunneriath, D., Karas, V., \& Das, T. K. 2013b, A\&A, 555, A97

Davidson, J. A., Werner, M. W., Wu, X., et al. 1992, ApJ, 387, 189

Dexter, J., Agol, E., Fragile, P. C., \& McKinney, J. C. 2010, ApJ, 717, 1092

Eckart, A., \& Genzel, R. 1996, Nature, 383, 415

Eckart, A., Schödel, R., \& Straubmeier, C. 2005, The Black Hole at the Center of the Milky Way (London: Imperial College Press)

Eckart, A., Britzen, S., Horrobin, M., et al. 2013a, in Nuclei of Seyfert Galaxies and QSOs, Proc. Sci., 004

Eckart, A., Horrobin, M., Britzen, S., et al. 2013b [arXiv: 1311.2753]

Eckart, A., Mužić, K., Yazici, S., et al. 2013c, A\&A, 551, A18

Field, G. B. 1965, ApJ, 142, 531

Frank, J., King, A., \& Raine, D. J. 2002, Accretion Power in Astrophysics: 3nd edn. (Cambridge: Cambridge University Press)
Genzel, R., Eisenhauer, F., \& Gillessen, S. 2010, Rev. Mod. Phys., 82, 3121 Ghez, A. M., Klein, B. L., Morris, M., \& Becklin, E. E. 1998, ApJ, 509, 678 Gillessen, S., Genzel, R., Fritz, T. K., et al. 2012, Nature, 481, 51

Gillessen, S., Genzel, R., Fritz, T. K., et al. 2013a, ApJ, 763, 78

Gillessen, S., Genzel, R., Fritz, T. K., et al. 2013b, ApJ, 774, 44

Gould, A., \& Quillen, A. C. 2003, ApJ, 592, 935

Hills, J. G. 1988, Nature, 331, 687

Hönig, S. F., \& Kishimoto, M. 2010, A\&A, 523, A27

Hopman, C., \& Alexander, T. 2006, ApJ, 645, 1152

Innanen, K. A. 1979, AJ, 84, 960

Keppens, R., van Marle, A. J., \& Meliani, Z. 2012, in Numerical Modeling of Space Plasma Flows, eds. N. V. Pogorelov, J. A. Font, E. Audit, \& G. P. Zank, ASP Conf. Ser., 459, 73

Kishimoto, M., Hönig, S. F., Antonucci, R., et al. 2011, A\&A, 536, A78

Krishna Swamy, K. S. 2005, Dust in the Universe: Similarities and Differences, World Scientific Series in Astronomy and Astrophysics, 7 (Singapore: World Scientific Publishing)

Krolik, J. H. 1999, Active Galactic Nuclei: From the Central Black Hole to the Galactic Environment (Princeton: Princeton University Press)

Krolik, J. H., \& Begelman, M. C. 1988, ApJ, 329, 702

Kunneriath, D., Eckart, A., Vogel, S. N., et al. 2012, A\&A, 538, A127

Kwok, S. 1975, ApJ, 198, 583

Levison, H. F., \& Duncan, M. J. 1994, Icarus, 108, 18

Löckmann, U., Baumgardt, H., \& Kroupa, P. 2008, ApJ, 683, L151

MacGregor, K. B., \& Stencel, R. E. 1992, ApJ, 397, 644

Meier, D. L. 2012, Black Hole Astrophysics: The Engine Paradigm (Berlin: Springer)

Melia, F. 2007, The Galactic Supermassive Black Hole (Princeton: University Press)

Moultaka, J., Eckart, A., Schödel, R., Viehmann, T., \& Najarro, F. 2005, A\&A, 443, 163

Murray-Clay, R. A., \& Loeb, A. 2012, Nature Commun., 3

Mužić, K., Eckart, A., Schödel, R., et al. 2010, A\&A, 521, A13

Narayan, R., \& McClintock, J. E. 2008, New Astron. Rev., 51, 733

Paumard, T., Maillard, J.-P., \& Morris, M. 2005, in Growing Black Holes: Accretion in a Cosmological Context, eds. A. Merloni, S. Nayakshin, \& R. A. Sunyaev (Berlin: Springer), 197

Perets, H. B., Hopman, C., \& Alexander, T. 2007, ApJ, 656, 709

Perets, H. B., \& Murray-Clay, R. A. 2011, ApJ, 733, 56

Phifer, K., Do, T., Meyer, L., et al. 2013, ApJ, 773, L13

Psaltis, D. 2012, ApJ, 759, 130

Quataert, E. 2004, ApJ, 613, 322

Sądowski, A., Narayan, R., Sironi, L., \& Özel, F. 2013, MNRAS, 433, 2165

Scoville, N., \& Burkert, A. 2013, ApJ, 768, 108

Shakura, N. I., \& Sunyaev, R. A. 1973, A\&A, 24, 337

Shcherbakov, R. V. 2014, ApJ, 783, 31

Šubr, L., \& Karas, V. 1999, A\&A, 352, 452

Šubr, L., \& Karas, V. 2005, A\&A, 433, 405

Urry, C. M., \& Padovani, P. 1995, PASP, 107, 803

Valencia-S, M., Bursa, M., Karssen, G., et al. 2012, J. Phys. Conf. Ser., 372, 012073

van Marle, A. J., Meliani, Z., Keppens, R., \& Decin, L. 2011, ApJ, 734, L26

Vilkoviskij, E. Y., \& Czerny, B. 2002, A\&A, 387, 804

Vladimirov, S. V., Ostrikov, K., \& Samarian, A. A. 2005, Physics and Applications of Complex Plasmas (London: Imperial College Press)

Wilkin, F. P. 1996, ApJ, 459, L31

Zhao, J.-H., Blundell, R., Moran, J. M., et al. 2010, ApJ, 723, 1097

Zurek, W. H., Siemiginowska, A., \& Colgate, S. A. 1994, ApJ, 434, 46 